\title{
Führung zwischen Tradition und Wandel
}

Welcher Führungsstil ist der richtige? Und gibt es überhaupt den richtigen Führungsstil?

Die vorangegangenen Kapitel haben gezeigt, dass es umfangreiche Anforderungen an und Herausforderungen für Führung gibt. Lassen sich diese Anforderungen tatsächlich alle in einem Führungsstil vereinen? Man muss kein Experte sein, um diese Frage mit „Nein“ zu beantworten. Wenn es aber unterschiedliche Führungsstile gibt, wie grenzen sich diese voneinander $a b$ ?

Eberhardt und Majkovic (2015) benennen in ihrer explorativen Studie gleich neun Führungsstile. Die Tab. 4.1, 4.2 und 4.3 geben einen kurzen Überblick zu deren zentralen Merkmalen.

Philipp (2010) verweist im Zusammenhang mit Führungsstilen auf eine Langzeitstudie der Harvard University. Danach prägen die eigenen Führungsvorbilder das spätere Verhalten einer Führungskraft und den damit verbundenen Führungsstil nachdrücklich. Erlebte der/die Arbeitnehmer*in eine Führungskraft, die ihn/sie forderte und förderte und führte dies zum Erfolg, nahm er/sie später in Führungsverantwortung eine ähnliche Grundhaltung ein. 
Tab. 4.1 Personal, Co- und Liquid Leadership

\begin{tabular}{|c|c|c|}
\hline Personal Leadership & Co-Leadership & Liquid Leadership \\
\hline $\begin{array}{l}\text { Führung erfordert ein } \\
\text { individualisiertes } \\
\text { Vorgehen; } \\
\text { Mitarbeitende sind } \\
\text { einzigartige, nicht } \\
\text { austauschbare Individuen; } \\
\text { Sicherung von Talenten } \\
\text { durch Fokus auf das } \\
\text { Individuum; } \\
\text { Jedes Individuum bringt } \\
\text { sein eigenes ,Paket mit“; } \\
\text { Führungsperson in der } \\
\text { Rolle eines/einer } \\
\text { Direktors/Direktorin bzw. } \\
\text { Coach; } \\
\text { Mitarbeiter*innenentwick- } \\
\text { lung nimmt an Bedeutung } \\
\text { zu. }\end{array}$ & $\begin{array}{l}\text { Zunehmende } \\
\text { Interdependenzen } \\
\text { erfordern das } \\
\text { Aushandeln } \\
\text { gemeinsamer Benefits; } \\
\text { Fähigkeit, Beziehungen } \\
\text { und ein heterogenes } \\
\text { Netzwerk aufzubauen; } \\
\text { Flexible Anpassung des } \\
\text { Führungsstils; } \\
\text { Unterschiedliche } \\
\text { Personen mit den } \\
\text { essenziellen } \\
\text { Informationen } \\
\text { zusammenführen und } \\
\text { das Gesamtbild } \\
\text { erhalten. }\end{array}$ & $\begin{array}{l}\text { Führungspersonen sollten } \\
\text { Orientierung geben; } \\
\text { Rolle als Mentor*in/Navigator*in, } \\
\text { die/der den Mitarbeitenden hilft, } \\
\text { Netzwerke aufzubauen; } \\
\text { Klare Ziele und Erwartungen } \\
\text { definieren; } \\
\text { Mit dem Team eine gemeinsame } \\
\text { Identität, Vision und Zielsetzungen } \\
\text { entwickeln; } \\
\text { Eine Zunahme von Online- } \\
\text { Beziehungen führt zu einem } \\
\text { höheren Bedürfnis, persönliche } \\
\text { Begegnungen herzustellen; } \\
\text { Führungskräfte schaffen bei } \\
\text { persönlichen Begegnungen positive } \\
\text { Interaktionen und positive } \\
\text { gemeinsame Erfahrungen. }\end{array}$ \\
\hline
\end{tabular}

Eigene Darstellung in Anlehnung an Eberhardt und Majkovic (2015)

Tab. 4.2 Complexity, Age- und Gender-related Leadership

\begin{tabular}{|c|c|c|}
\hline Complexity Leadership & Age-related Leadership & Gender-related Leadership \\
\hline $\begin{array}{l}\text { Mitarbeitenden die } \\
\text { Möglichkeit geben, neue } \\
\text { Ideen auszuprobieren. } \\
\text { „Try, fail and restart“ } \\
\text { (jedoch ressourcen- } \\
\text { schonend); } \\
\text { Wie Wissenschaftler*innen } \\
\text { denken: Intellektuelle } \\
\text { Neugier als } \\
\text { Entwicklungsmotor; } \\
\text { Mitarbeitende dazu } \\
\text { befähigen, zielorientiertere } \\
\text { Fragen zu stellen; } \\
\text { Intensive } \\
\text { Auseinandersetzung mit } \\
\text { den Resultaten der } \\
\text { Führungsentscheidungen; } \\
\text { Regelmäßige Adaption von } \\
\text { Strategien. }\end{array}$ & $\begin{array}{l}\text { Führungspersonen sollten } \\
\text { fähig sein, die Stärken von } \\
\text { unterschiedlichen } \\
\text { Altersgruppen zu nutzen; } \\
\text { Signifikante Altersunter- } \\
\text { schiede zwischen den } \\
\text { Führungspersonen und } \\
\text { den Mitarbeitenden } \\
\text { bedingen } \\
\text { Erwartungsklärungen und } \\
\text { Zieldefinitionen; } \\
\text { Stärken und Schwächen } \\
\text { der Teams identifizieren; } \\
\text { Kooperative und positive } \\
\text { Begegnungsräume für } \\
\text { unterschiedliche } \\
\text { Altersgruppen schaffen. }\end{array}$ & $\begin{array}{l}\text { Geschlechterneutrales Vorgehen } \\
\text { bei der Identifikation von Talent; } \\
\text { Gleichstellungsinitiativen in die } \\
\text { Agenda von Führungspersonen } \\
\text { integrieren anstelle von } \\
\text { Delegation an Verantwortliche } \\
\text { für Diversität; } \\
\text { Mentoring Programme für } \\
\text { Frauen; } \\
\text { Frauen, die in Top- } \\
\text { Managementpositionen } \\
\text { ankommen wollen, sollten fähig } \\
\text { sein, ihr Netzwerk zu } \\
\text { mobilisieren; } \\
\text { Authentizität, Moralität, und } \\
\text { Ehrbarkeit gelten als } \\
\text { erstrebenswerte } \\
\text { Führungstugenden unabhängig } \\
\text { vom Geschlecht der } \\
\text { Führungsperson. }\end{array}$ \\
\hline
\end{tabular}

Eigene Darstellung in Anlehnung an Eberhardt und Majkovic (2015) 
Tab. 4.3 Change, Intercultural und Sustainable Leadership

\begin{tabular}{l|l|l}
\hline Change Leadership & Intercultural Leadership & Sustainable Leadership \\
\hline Eine Prognose für die & Die kulturelle Denkart & Führung agiert als Vorbild in den \\
Zukunft entwickeln; & weiterentwickeln und den & Themen der sozialen \\
Führungskräfte & eigenen kulturellen Horizont & Verantwortung und der \\
sollten sich raschen & erweitern; & Nachhaltigkeit; \\
ökonomischen, & Sich mit den wesentlichen & Systemdenken verbessern und sich \\
nationalen und & kulturellen Bräuchen ihrer & der Auswirkungen der \\
politischen & Mitarbeitenden bekannt & Entscheidungen außerhalb der \\
Veränderungen & machen; & Organisationsgrenzen bewusster \\
anpassen und ihre & Interkulturelle Kompetenzen & werden; \\
Führungsstrategien & im Ausland erlernen; & Die Implikationen der \\
entsprechend & Interkulturellen Austausch & Führungsentscheidungen auf \\
adaptieren; & unterstützen & interne und externe Stakeholder \\
Vernetztes Denken. & z. B. Auslandstätigkeiten); & abschätzen lernen; \\
& Interkulturelle Teams & Soziale Verantwortung und \\
fördern. & Rentabilität in ein Gleichgewicht \\
& & bringen. \\
\hline
\end{tabular}

Eigene Darstellung in Anlehnung an Eberhardt und Majkovic (2015)

Unabhängig von Führungsstilen zeigt Philipp einen 9-fachen Pfad der ganzheitlichen Führung auf:

1. Bei sich selbst beginnen: Selbstverantwortung, (De-)Mut, Ethik, Disziplin, Entwicklung;

2. Werte und Respekt: Fairness, Fürsorge, Respekt, Leistungswille, Integrität;

3. Visionärer Realitätssinn: Pro-aktiv, Beharrlichkeit, Vision, Mission, Aktionsplan;

4. Fokussieren: Transparenz, Prioritäten setzen, Konzentration der Kräfte;

5. Planung und Management: Resultate erzielen, Probleme lösen, effektive Meetings;

6. Im Sinne der Sache handeln: Die Aufgabe ist wichtiger als das eigene Ego, Authentizität;

7. Kommunikation: Empathie, Mitgefühl, zuhören, verstehen, verstanden werden;

8. Kooperation: Win-Win, Unterschiedlichkeit, Teams, Synergie;

9. Sich selbst erneuern: Kompetenz-Netzwerke, evolutionäres Lernen, Lebensbalance finden und halten. 
Er sieht diesen Pfad als Entwicklungsstufen, bei dem sich die Stufen 1-5 und 9 tendenziell mit sich und dem direkten Umfeld beschäftigen, während die Stufen 6-8 mehr die Interaktion mit anderen im Fokus haben.

Führungsstile sieht er lediglich als Orientierung. Sie sollen dazu einladen, sich selbst zu reflektieren:

- Wo erkenne ich mich am ehesten wieder?

- Wo bin ich authentisch?

- Wohin möchte ich mich weiterentwickeln?

Wenn im Folgenden einzelne, aus heutiger Sicht moderne Führungsstile beschrieben werden, soll diese Einladung erneuert werden. Verschaffen Sie sich gerne einen Überblick und gewinnen Sie einen Eindruck davon, welche(r) Führungsstil(e) Sie am ehesten anspricht/ansprechen.

Als ersten Einstieg, gibt Abb. 4.1 einen groben Überblick über populäre Führungsstile der heutigen Zeit.

\subsection{Transformationale Führung}

Kaum ein Führungsstil wird in den vergangenen Jahren so als idealtypisch angepriesen wie die transformationale Führung. Gleichzeitig löst kaum ein Führungsstil mehr Verunsicherung bei erfahrenen Führungskräften aus, geht mit ihm doch häufig die Forderung nach mehr charismatischer Führung einher.

\section{Warum braucht es überhaupt eine transformationale Führung?}

Sprenger (2017) verweist auf die Notwendigkeit, sich künftig verstärkt mit dem Entwurf von Krisen- und Zukunftsszenarien zu beschäftigen. Als zukunftsfähig bezeichnet er Firmen, in denen wahrscheinliche und unwahrscheinliche Szenarien diskutiert werden (z. B. in Open-Space-Konferenzen) und Meinungsvielfalt zur notwendigen Redundanz führt. Ebenso sieht er Unternehmen als gut vorbereitet, die Eigensinn und Widerspruchsgeist, Gehorsam und Konformität vorziehen.

Sprenger betont, dass es einer Ambiguitätstoleranz bedarf, die Führung massiv fordern wird. Das bedeutet, dass Führung auf Prinzipien verzichten und stattdessen 


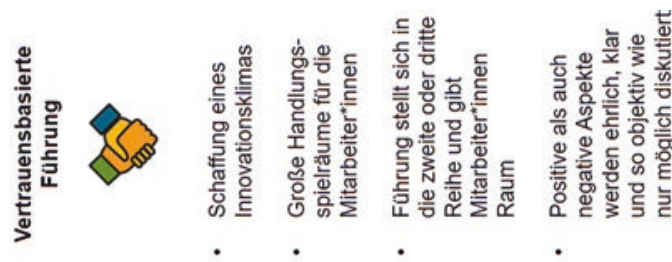

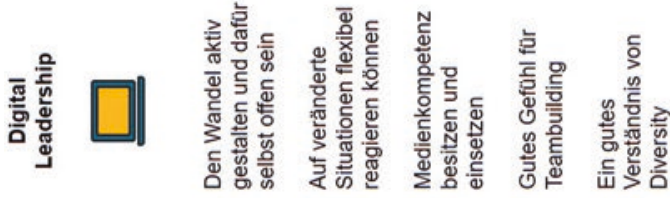

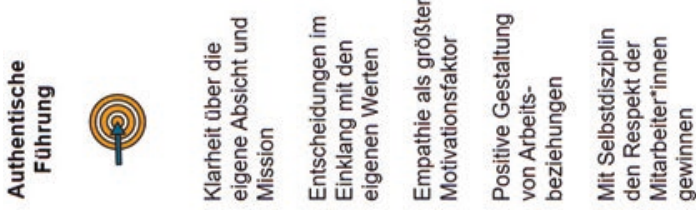

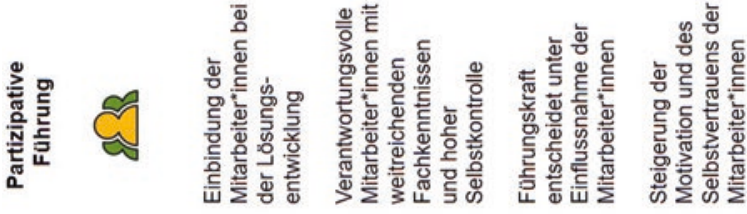
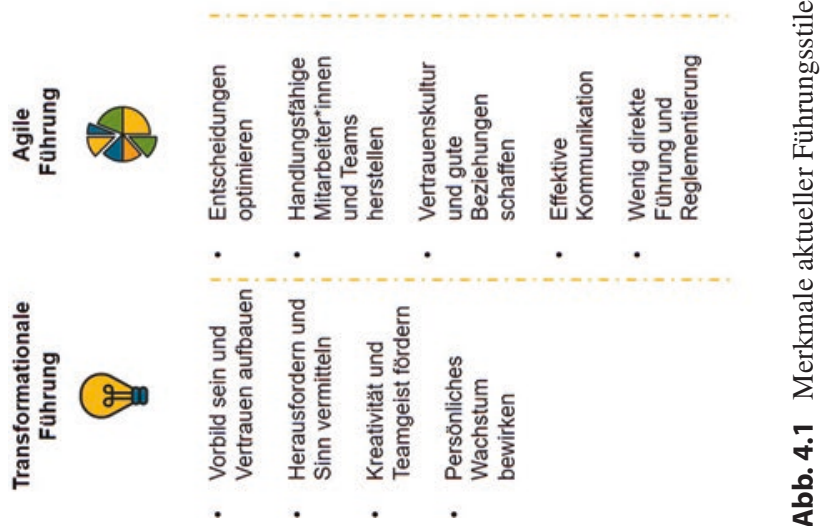
Unklarheit, Mehrdeutigkeiten und Paradoxien mögen und darin Chancen statt Risiken erkennen muss.

Am Beispiel von Digitalisierung zeigt er Ambiguitäten auf, die er an sieben Unterschieden in Leitung und Führung festmacht:

1. Physisch/virtuell: Kommunikation und Kooperation verlagern sich in den virtuellen Raum. Dies bedingt, kommunikationsfördernde Architekturen und Gehaltssysteme neu zu denken.

2. Innen/außen: Die Unternehmensgrenzen verschwimmen. Zeitarbeit, Befristungen und Digitalnomadentum machen das Identitätsstiftende von Unternehmensgrenzen bewusst.

3. Kurzfristig/langfristig: Insgesamt wird die Instabilität innerhalb und außerhalb der Unternehmen zunehmen. Langfristige Planung ist schwierig, lineare Führung mit Zielen, starre Budgetprozesse und langatmige Reportings gehören der Vergangenheit an.

4. Privat/beruflich: Die Vorstellungen von Karriere und Sinnsuche vervielfältigen sich. Die neue Währung ist Zeit, Gesundheit und Sinn. Führung wird sich daher vom „One size fits all“" kulturell wie arbeitsorganisatorisch verabschieden.

5. Vertikal/horizontal: Hierarchie, Netzwerk, Co-Leadership und Projekte überlagern und unterlaufen sich flexibel. Führung wird indirekter. Es gilt nicht mehr, einzelne Mitarbeiter*innen zu optimieren, sondern Netze zu flechten und Aufmerksamkeit zu kanalisieren. Fachliche Überlegenheit wird Führung nur noch selten legitimieren. Auch ein Drohen funktioniert bei flexiblen Spezialist*innen nicht.

6. Individuell/kollektiv: Es gilt digitale Eliten zu einem Kollektiv zu vereinen.

7. Ökonomisch/moralisch: Theoretisch lassen sich bereits heute technische Möglichkeiten nutzen, mit soziometrischen Informationen das Verhalten des Mitarbeiters/der Mitarbeiterin (,Wo ist er? Was tut er? Wohin geht er?“) bis hin zu seiner Stimmung zu kontrollieren. Ethisch führt dies zu der Frage, wie weit man gehen sollte und darf.

Sprenger resümiert:

Es ist eine Herausforderung für viele Unternehmen, eine veränderungsbereite und -fähige Organisation zu schaffen. Man hat ja alle zum Gegner, die aus dem Herkömmlichen ihre Vorteile ziehen. Und das sind vorrangig die Führungskräfte selbst. Aber es hilft nichts, ungestörtes Arbeiten ist der sichere Weg ins Verderben. Wenn man das Unternehmen zukunftsfähig machen will, dann muss man es transformationsfähig machen. 
Bedenkt man, dass der transformationale Führungsstil bereits auf das Jahr 1978 zurïckgeht, so ist umso verwunderlicher, dass dieser Ansatz mit großer Zeitverzögerung erst im aktuellen Umfeld seine Renaissance erlebt.

Der Politologe James MacGregor Burns (1978) differenzierte im Rahmen einer Untersuchung über Führung im politischen Kontext zwei Arten von Führungskräften.

- Diejenigen, die Gefolgschaft dadurch erreichen, dass sie Vorteile aller Art (Belohnung) gegen die Leistung der Geführten tauschen (transaktional).

- Diejenigen, die durch eine wechselseitige Pflichtgemeinschaft mit den Geführten deren Motivation und Zusammenhalt kontinuierlich steigern (transformierend).

Während Burns davon ausging, dass entweder der transaktionale oder der transformierende Führungsstil zur Anwendung kommt, weitete Bass (1985) dies bei seiner Anwendung auf Führung im Unternehmen auf einen zweidimensionalen Ansatz aus. Nach seiner Meinung existieren beide Führungsstile unabhängig voneinander und können sowohl allein als auch gleichzeitig zur Anwendung kommen.

Bei der transaktionalen Führung orientiert sich die Führungskraft an Zielen als auch am bestehenden Werteniveau und der Persönlichkeitsstruktur und den damit einhergehenden Erwartungen der Geführten.

In Zeiten nachhaltiger Unsicherheit und Veränderung braucht es jedoch mehr als das. Ausgehend von den Zielen, Werten und Wünschen der Mitarbeiter*innen gilt es, diese tief greifend und dauerhaft zu wandeln und auf eine höhere Stufe zu transformieren.

Bass und Avolio (1990) nennen die folgenden Merkmale einer transformationaler Führung:

- Vorbildfunktion, die Bewunderung, Respekt und Vertrauen erzeugt;

- Werte und Ideale werden vermittelt;

- Charismatisches Verhalten;

- Inspirierende und herausfordernde Vision der Zukunft zeichnen;

- Bedeutungsvolle Ziele setzen;

- Hohe Leistungserwartungen zeigen;

- Anregung neuer Ideen und Denkweisen;

- Kritisches Hinterfragen des Status quo;

- Kreativität und Innovationen fördern;

- Persönliche Unterstützung gemäß der individuellen Bedürfnisse und Wünsche der Geführten;

- Weiterentwicklung der Geführten. 
Ein Führungsverhalten, das mit einer charismatischen Attribuierung der Führungsperson einhergeht, bringt positive Auswirkungen auf Motivation und Leistung der Mitarbeiter*innen hervor, die ansonsten nicht oder kaum zu erzielen wären.

Furtner (2016) nennt die folgenden positiven Effekte einer transformationalen Führung:

- Die individuelle Leistung, die Gruppenleistung und die Gesamtleistung der Organisation,

- die Arbeitszufriedenheit und Arbeitsmotivation der Geführten,

- die Effektivität der Geführten,

- die freiwilligen Mehrleistungen der Geführten,

- die Unterstützung der Arbeitskolleg*innen,

- die Kreativität und Innovationsfähigkeit der Geführten,

- die organisationale Innovation und das organisationale Lernen,

- die Selbstwirksamkeit (den Glauben an die eigenen Fähigkeiten) der Geführten,

- das emotionale Vertrauen der Geführten,

- die Identifikation und die (moralische) Verpflichtung der Geführten zu ihrer

- Führungskraft und Organisation,

- die Hoffnung, den Optimismus, die Ausdauer und die Widerstandsfähigkeit

- der Geführten,

- das emotionale Wohlbefinden der Geführten.

Wenn Charisma jedoch eine zentrale Bedeutung bei diesem Führungsstil einnimmt, zieht dies unweigerlich die Frage nach sich, ob diese Eigenschaft tatsächlich erlernbar ist.

Und wodurch zeichnet sich eine charismatische Führung überhaupt aus?

Conger und Kanungo (1998) formulieren ein Modell, indem sie das charismatische Führungsverhalten betrachten. Es zeichnet sich demnach folgendermaßen aus:

- Kommunikation einer Vision: Die Führungskraft bietet inspirierende Ideen und Visionen und kann diese beeindruckend präsentieren.

- Politisches Gespür: Die Führungskraft erkennt rechtzeitig Gefahren und Risiken aber auch Chancen im sozialen und technischen Umfeld.

- Unkonventionelles Verhalten: Die Führungskraft überrascht durch ungewöhnliche Maßnahmen und Abweichung von gewohnten Abläufen.

- Persönliche Risikobereitschaft: Die Führungskraft nimmt persönliche Risiken in Kauf und scheut keinen persönlichen Aufwand. 
- Sensibilität für Bedürfnisse der Mitarbeiter*innen: Die Führungskraft zeigt Interesse für die Bedürfnisse der Mitarbeiter*innen und behandelt diese mit Respekt.

- Empowerment: Die Führungskraft gestaltet und delegiert verantwortungsvolle Aufgaben an die Mitarbeiter*innen.

Nach Furtner (2016) haben Persönlichkeitseigenschaften und Motive einen Einfluss darauf, ob eine Führungskraft als mehr oder weniger charismatisch wahrgenommen wird:

Eine introvertierte (in sich gekehrte) Führungskraft wird es schwieriger haben, tatsächlich von ihren Geführten als charismatisch wahrgenommen zu werden. Es ist jedoch nicht unmöglich: Durch intensives Training können die spezifischen Wirkmechanismen (z. B. Formulierung einer Vision, rhetorische Fähigkeiten) des Charismas entwickelt werden.

Wichtig ist an diesem Punkt immer, dass die Führungskraft authentisch ist und nicht in eine unnatürliche Rolle fällt. Dies würde den Wirkmechanismus des Charismas ,zerstören“.

Ebenso zerstörerisch für das soziale Umfeld kann es sein, wenn eine Führungskraft Charisma auf Basis sogenannter „dunkler Eigenschaften“ entwickelt. Antrieb ist dann ein Egoismus, der dem absoluten Selbstzweck dient (Abb. 4.2).

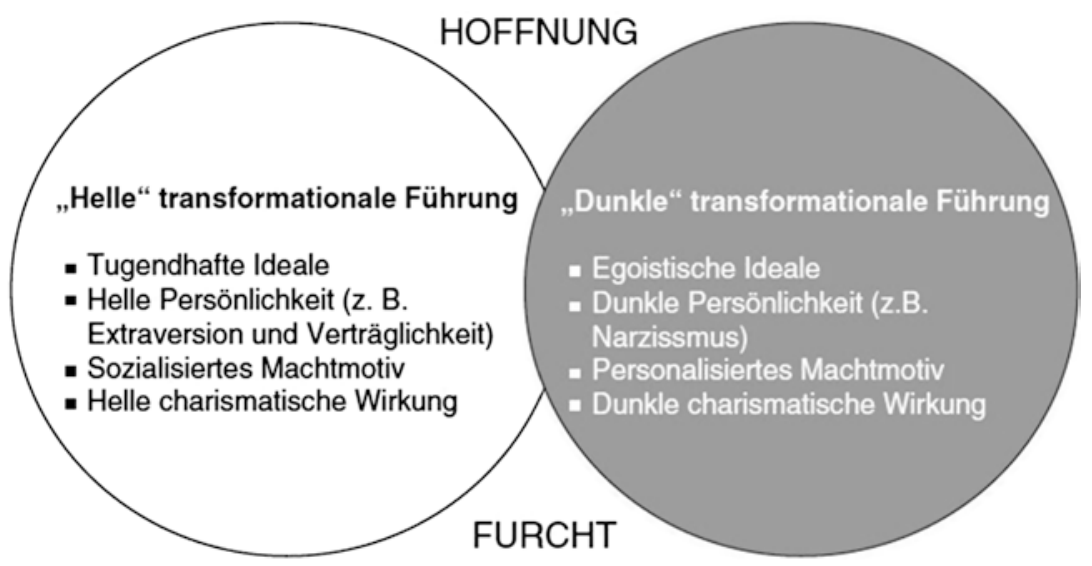

Abb. 4.2 Motivationale Wirkkraft des Charismas 
Eine relativ selbstlose und soziale Führungskraft zeigt im Sinne eines hellen Charismas einen reduzierten Ego-Fokus. Sie verfolgt höhere Ideale und Ziele und handelt nachhaltig für ihr Umfeld. Dadurch ergeben sich wiederum langfristig positive Konsequenzen für das soziale Umfeld.

Harms und Credé (2010) nennen eine weitere Einflussgröße, die das Charisma und die transformationale Führung möglicherweise begünstigt: Die emotionale Intelligenz.

Emotional intelligenten Führungskräften gelingt es, die eigenen Emotionen und Gefühle richtig wahrzunehmen. Ebenso sind sie in der Lage, die Emotionen und Gefühle von anderen Menschen in eine bestimmte Richtung zu lenken. Sie verfügen über das entsprechende Einfühlungsvermögen (Empathie), um die Emotionen und Gefühle von anderen Menschen zu verstehen und positiv zu beeinflussen. Auch wenn die emotionale Intelligenz förderlich für das Auftreten von transformationaler Führung sein kann, ist sie jedoch nicht der alles entscheidende Faktor.

\section{Praxisbeispiel}

Ich arbeite in Einzelcoachings gerne mit Persönlichkeits-/Motivstrukturanalysen (vgl. Abschn. 5.2). Dies deshalb, um schneller einen Zugang zu den Möglichkeiten und Entwicklungsfeldern von Führungskräften zu erhalten. In diesem Zusammenhang zeigt sich, dass es nicht jedem Menschen in die Wiege gelegt ist, sich mit den eigenen Emotionen und erst recht der emotionalen Welt anderer adäquat auseinander zu setzen. Man spürt regelrecht das Unbehagen, wenn sich das Gespräch in diese Richtung neigt. Die Führungskräfte versuchen dann auszuweichen, zu bagatellisieren oder zu theoretisieren. Teilweise suchen sie Gesprächsanleitungen und Tools, wie sie emotionalen Situationen mit Ihren Mitarbeiter*innen besser gerecht werden können. In seltenen Fällen möchten sie dies komplett vermeiden und nehmen sich als Vorgesetzte das Recht, Emotionalität aus dem Berufskontext auszuklammern. Wird Emotionalität Bestandteil des Coachings, kann es vorkommen, dass sie das Coaching nicht weiterführen, um sich der für sie unangenehmen Situation zu entziehen.

Dennoch müssen dies keine schlechten Führungskräfte sein. Im Gegenteil: Sie begeistern ihre Mitarbeiter*innen über ihr Engagement und/oder ihre hohe Expertise im Fachlichen und Methodischen. Sie sind innovativ und motiviert und vermitteln dies ihren Mitarbeiter*innen Tag für Tag. Sie fordern ihre Mitarbeiter*innen zu Höchstleistungen und geben ihnen Stütze und Orientierung, teilweise bis über die eigenen Belastungsgrenzen hinaus. Nicht immer sind sie extrovertiert, sondern eher von stiller und zurückgenommener Art, aber dennoch hoch respektiert bei den Teams, die sie verantworten. 
Ihre Herausforderungen liegen in der Konfliktbewältigung. Bei der Selbstreflexion fehlen ihnen wichtige innere Zugangswege, um sich selbst und das Miteinander mit anderen besser einordnen zu können. Sie spüren, dass es ihnen schwerer fällt, in Konfliktsituationen an das Kernthema des Mitarbeiters/der Mitarbeiterin heranzukommen. Es gelingt ihnen nicht, sich auf deren Bedürfnisebene einzulassen, erst recht, wenn es um die Verletzung emotionaler Bedürfnisse geht.

Nun erleben sie in zunehmendem Maße, dass man ihnen durch neue Führungsstile, wie dem transformationalen, vorhält, sich ihren Schwächen stellen zu müssen. Mehr Charisma, mehr Emotionalität, mehr Psychologie und Soziologie statt Betriebswirtschaft, Jura, Mathematik oder IT. Wie so häufig führt es eher zu Verunsicherung und Ablehnung, wenn bei allen Führungskräften versucht wird, im Zuge einer „Das-machen-wir-jetzt-künftig-alle-gleich-Forderung“ mit der Heckenschere Konformismus zu erzeugen. An dieser Stelle spreche ich mich daher deutlich für Diversität und einen genauen Blick dafür aus, was es im Kontext der spezifischen Führungssituation und Aufgabe wirklich braucht. Kap. 5 wird dies deutlicher thematisieren.

Dennoch soll dies kein Alibi sein, sich bei der Reflexion über die eigenen Führungsaufgaben und Grundhaltungen mit den dafür erforderlichen Sachverhalten nicht auseinandersetzen zu müssen.

\section{Fazit aus diesem Abschnitt}

Die Merkmale des bereits über 40 Jahre alten transformationalen Führungsstils rücken aktuell in den Fokus der Führungspraxis. Führung zeichnet sich in diesem Kontext durch Charisma und das Einbringen einer emotionalen Komponente (im Sinne der Selbstreflexion, aber vor allem der Auseinandersetzung mit den Emotionen und Gefühlen von Mitarbeiter*innen) aus.

\subsection{Authentische, vertrauensbasierte Führung}

Ein Führungsstil, der auf reinem Vertrauen aufbaut? Gerade jetzt? Gerade in Krisenzeiten in denen Regulatorik und die damit verbundene Kontrollnotwendigkeit in den Finanzhäusern dominiert? Manche werden diese Forderung als unrealistische Sozialromantik abtun.

Dass aber genau dies funktioniert, zeigt Kronawitter (2013) in Zusammenarbeit mit der Ruhr-Universität Bochum. Seine auf Vertrauen ausgerichtete Unterneh- 
mens- und Führungskultur führt zu einem signifikant höheren Commitment der Mitarbeiter*innen seiner Raiffeisenbank, als dies bei zwei Referenzgruppen der Fall ist. Zudem zeigen alle Werte der in der Mitarbeiterbefragung verwendeten Skalen Bereichsklima (Vorgesetztenverhalten, Zusammengehörigkeitsgefühl), Unternehmensklima (Motivation der Belegschaft, Stimmung im Unternehmen), Unternehmensstruktur (kurze Entscheidungswege, klare Regeln, Kooperation), Kontinuität (langfristige Planung, Stabilität, Krisensicherheit), Tätigkeitsanreiz (Anerkennung, angemessene Bezahlung), berufliche Entfaltung (Aufstiegs- und Weiterentwicklungsmöglichkeiten), Tätigkeit (Abwechslung, Vollständigkeit der Arbeit, eigene Planung) und Arbeitsbedingungen (Möglichkeit zur Erholung in Pausen, Flexibilität der Arbeitszeit) überdurchschnittlich bessere Ausprägungen.

Betrachtet man die Merkmale, die der Vorstand der Genossenschaftsbank einer vertrauensbasierten Führung zuordnet, sind dies

- ein Verzicht auf individuelle Vorgaben und Produktkampagnen, stattdessen eine hohe Handlungs- und Entscheidungsfreiheit der Mitarbeiter*innen,

- eine Abkehr von Provisionen und variablen Vergütungen, stattdessen marktgerechte Gehälter,

- ausreichende Konditionsspielräume im Kundengeschäft je Berater*innen, mit unternehmerischer Verantwortung für einen adäquaten Deckungsbeitrag je Kund*in,

- Vollmachten für den Einkauf von Sachmitteln und ausreichende Befugnisse zur direkten Klärung von Reklamationen und Beschwerden je Mitarbeiter*in,

- eine offene Krisenkommunikation, statt Intransparenz und Schüren des Flurfunks,

- eine flache Hierarchie und effiziente Entscheidungswege mit einer von Unmittelbarkeit und Direktheit geprägten Kommunikation,

- eine Fehlerkultur, die nicht darauf abzielt, Schuldige zu finden,

- Teams mit verteilten Aufgaben, die sich ergänzende Kompetenzen gemeinsam nutzen, sowie Beteiligung der Mitarbeiter*innen an Projekten,

- konsequentes Handeln mit ebenso konsequenten Reaktionen bei Nichteinhaltung der vereinbarten Spielregeln,

- Authentizität, d. h. wahrnehmbare Kommunikation und Leben grundsätzlicher Werte, ohne dabei die Normen eines Sozialverhaltens, die Spielregeln der Bank oder die gesetzlichen Vorschriften zu verletzen,

- persönlicher Austausch von Informationen, statt sich hinter E-Mails zu verstecken, 
- eindeutige Entscheidungen, statt quälender Konsens,

- der richtige Einsatz von Talenten und zielgerichtete Personalentwicklung,

- ein gerechtes Handeln aus innerer Überzeugung,

- Mitarbeiter*innen, die ihre freien Tage selbst verantworten,

- die Reaktivierung von Qualifikationen von beruflichen Wiedereinsteiger*innen und Sicherung von Qualifikationen vor dem Ruhestand,

- die Würdigung besonderer Anlässe (Persönliche Begegnungen wirken dabei stärker als digitale Kontakte),

- ein umfangreiches, beidseitiges Feedback an Mitarbeiter*innen und Führungskräfte,

- offene Türen bei den Führungskräften und

- hierarchiefreies Entstehen und Entfalten von Innovationen.

Dabei gilt, dass es sich bei den genannten Punkten nicht um eine Entwederoder-Auswahl handelt. Diese Aspekte wirken nur dann, wenn sie gemeinsam von allen in der Bank verinnerlicht und gelebt werden. Vertrauen lässt sich nicht einfordern, man kann es sich nur verdienen.

Ob die Unternehmenskultur dieser kleinen Bank auf größere Häuser übertragbar ist, bleibt offen.

Die Authentizität aller Beteiligten, vor allem jedoch der Führungskräfte, hat dabei eine zentrale Bedeutung.

Avolio et al. (2004) definieren authentisch Führende:

Those individuals who are deeply aware of how they think and behave and are perceived by others as being aware of their own and others' values/moral perspectives, knowledge, and strengths; aware of the context in which they operate; and who are confident, hopeful, optimistic, resilient, and of high moral character.

Gardner et al. (2005) wiederum betonen, dass Authentizität in der Führung über die Person des Führenden hinaus geht und gleichsam die Beziehung mit Geführten und Kolleg*innen umfasst. Abb. 4.3 greift diesen prozessualen Charakter auf.

Führende erreichen dabei zunächst Authentizität durch Selbst-Bewusstheit. Diese ist Voraussetzung für eine Selbstregulation. Hieraus entstehen authentische, vorbildhafte Handlungsweisen, die das Eingehen authentischer Beziehungen ermöglichen. Die Führungskraft versetzt sich selbst in die Lage, Authentizität bei anderen auszubilden. Hierzu erforderlich ist ein spezifischer organisationaler Rahmen, der förderlich auf die Führerenden-Geführten-Beziehung einwirkt. Damit schließt sich der Kreis zu den von Kronawitter aufgeführten Variablen. 


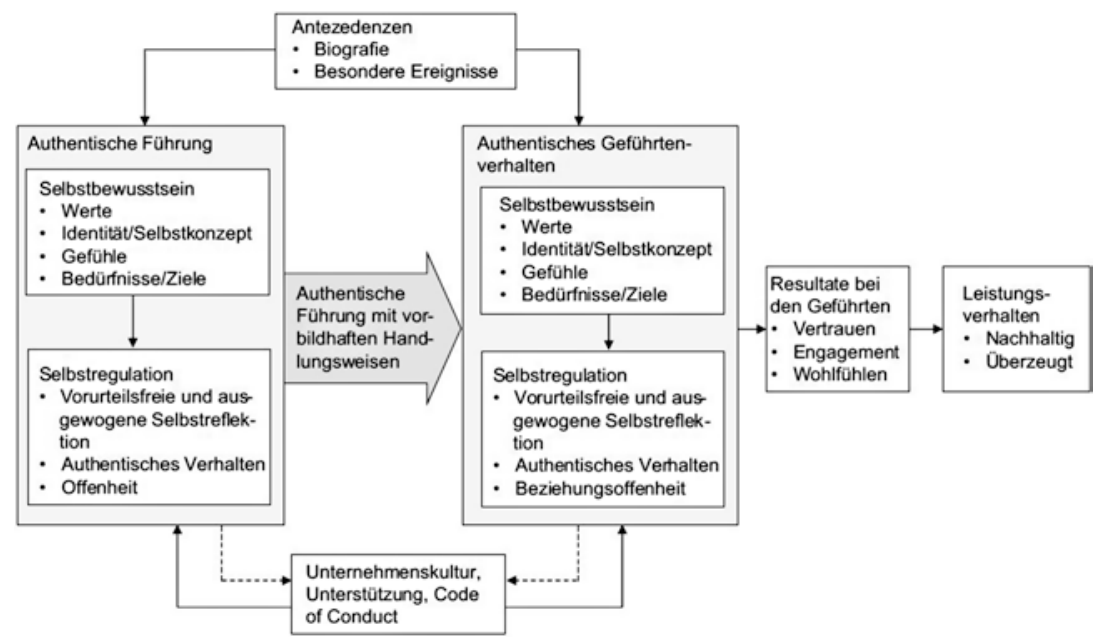

Abb. 4.3 Bezugsrahmen Authentische Führung

\section{Praxisbeispiel}

Wie schwierig es sich gestaltet, dass Führungskräfte, vor allem des mittleren Managements, authentisch bleiben, zeigt sich nicht selten, wenn es darum geht, gerade unangenehme Unternehmensentscheidungen mitzutragen. So stellen Führungskräfte in Führungsworkshops und -Coachings regelmäßig die Frage, wie offen sie ihren Mitarbeiter*innen gegenüber kommunizieren dürfen, dass sie nicht gänzlich hinter der Entscheidung des oberen Managements stehen.

Sie erleben einen intrapsychischen Konflikt, d. h. in ihnen entstehen unvereinbare, einander entgegengerichtete Handlungstendenzen (stehe ich kommentarlos zur Entscheidung des Managements und mache mich selbst unglaubwürdig oder vertrete ich meine Meinung und verhalte mich dadurch dem Management gegenüber illoyal). Dieses Erleben führt häufig zu emotionalen Spannungen.

Wenn Führung wirksam sein soll, müssen alle Führungsebenen eines Hauses an einem Strang und in dieselbe Richtung ziehen. Die Frage stellt sich somit nicht, ob die Filial-, Team- oder Abteilungsleitung die Entscheidung des Vorstandes oder der Bereichsleitung mitträgt. Vielmehr ist zu klären, welche Widersprüche und Fragen offen sind, die eine vollumfängliche Identifikation aktuell noch nicht möglich machen und wie diese ausgeräumt werden können.

Aber selbst wenn dieser Diskurs auf allen Führungsebenen geführt wurde, kann der innere Widerspruch bei der jeweiligen Führungskraft aufgrund diffe- 
renzierter Wertvorstellungen nach wie vor bestehen bleiben. Der Hauptansatz des Coaching liegt dann darin, eine vertretbare innere Grundhaltung zu entwickeln, die ein leichteres Handling des Widerspruchs möglich macht. Zusätzlich können gesichtswahrende Kommunikationsansätze in Richtung Mitarbeiter*innen erarbeitet werden.

Können intrapsychische Konflikte nicht gelöst werden, verliert die Führungskraft ihre Authentizität und damit Überzeugungs- und Strahlkraft auf die Mitarbeiter*innen. Kommt dies häufiger vor, sollte sich die betroffene Person im Extremfall zum eigenen Selbstschutz mit der Entscheidung auseinandersetzen, die Bank/Sparkasse zu verlassen oder ihre Rolle als Führungskraft aufzugeben.

\section{Fazit aus diesem Abschnitt}

Eine auf Vertrauen ausgerichtete Unternehmens- und Führungskultur führt zu einem signifikant höheren Commitment der Mitarbeiter*innen. Authentizität schafft Vertrauen, schließt ein sich-selbst-bewusst-werden mit ein und weitet sich auf die Beziehungen zu den Mitarbeiter*innen und Kolleg*innen aus. Gelingt es Führungskräften auf Dauer nicht, vorhandene innere Konflikte zur eigenen Grundhaltung im Vergleich zu den Zielvorstellungen des Unternehmens aufzulösen bzw. tragbar zu machen, setzten sie ihre Authentizität und damit Glaubwürdigkeit bei den Mitarbeiter*innen aufs Spiel.

\subsection{Digital Leadership}

Ist Digital Leadership neuer Wein in alten Schläuchen, wie es Wächter (2019) provokant formuliert? Im übertragenen Sinne verbirgt sich dahinter die Frage, ob sich die digitale Transformation eines Unternehmens überhaupt mit den erfahrenen Langzeit-Führungskräften vollziehen lässt. Die Antwort hierauf überlasse ich jedem selbst, ebenso wie es in der Selbstverantwortung einer jeden Führungskraft liegt, sich für die digitale Transformation fit zu machen und sich der hierfür erforderlichen Veränderung zu unterziehen.

Greift man die in Abschnitt 2.4 erläuterten Auswirkungen der Digitalisierung und die damit verbundene Veränderungsnotwendigkeit von Banken und Sparkassen auf, nimmt Führung jedenfalls den Platz einer zentralen Stellschraube ein.

Dies bestätigen auch Hays (2016) in ihrer Umfrage unter Führungskräften zu den Herausforderungen der digitalen Transformation für die Arbeitsorganisation in Deutschland, Österreich und der Schweiz. Demzufolge gaben $53 \%$ der Führungs- 
kräfte (bei 591 Befragten) an, eine der fünf größten Herausforderungen für die Arbeitsorganisation sei die nötige Anpassung der Führungskultur an flexible Arbeitsmodelle.

Aber was meint Digital Leadership genau?

Wagner (2018) zeigt vier Blickrichtungen für eine erste Begriffseingrenzung:

1. Führung mit digitalen Techniken (wörtliche Übersetzung)

2. Führung von digitalen Talenten (sinngemäße Übersetzung)

3. Digitale Marktführerschaft (sinngemäße Übersetzung)

4. Erfolgreiche Führung in Zeiten der digitalen Transformation (holistische Übersetzung)

Definition 4 bezieht die ersten beiden Interpretationen mit ein und bildet die Basis für die nachfolgenden Ausführungen.

Petry (2019) sieht bei Führungskräften in diesem Zusammenhang einerseits eine Treiber-, als auch Befähiger-Rolle für die Entwicklung eines digitalen Mindsets, und damit der Einstellung oder auch Grundhaltung zur Digitalisierung im Unternehmen. Dieses geht über das Verständnis der digitalen Kundenerfahrung hinaus, erstreckt sich auf das ganze Unternehmen und erfasst somit auch interne Prozesse und Vorgehensweisen.

Capgemini Invent (2015) geben einen Überblick darüber, wie sich Zusammenarbeit im digitalen Zeitalter verändert:

- Hyperconnectedness: Zusammenarbeit geschieht in Netzwerken aus Netzwerken, wobei es unmöglich wird, alle Verbindungen zu kontrollieren.

- Wissensmanagement: Statt individuelles Wissen wird kollektives Wissen sehr viel wichtiger - selbst erfahrene Expert*innen müssen auf Kenntnisse Dritter vertrauen.

- Flache Hierarchien: Virtuelle und kurzlebige Teams ersetzen bürokratische und hierarchische Strukturen.

- Kommunikation: Virtuelle Arbeitsplätze in unterschiedlichen Zeitzonen verändern die Kommunikation.

- Einfluss: Informelle Gruppen und Meinungsführer*innen können großen Einfluss auf den Erfolg einer Organisation haben.

- Arbeitsweisen: Innovationsprozesse sind von Interaktionen und nichtroutinierten Prozessen geprägt.

- Schnelligkeit: In allen Arbeitsbereichen wird der Innovations- und damit auch der Veränderungsrhythmus erhöht. 
Den digitalen Reifegrad von Führungskräften ordnen sie mit Hilfe eines Digital Leadership Assessments in einer Digital Leadership Matrix ein (vgl. Abb. 4.4).

Radermacher (2018) betont im Kontext einer zunehmenden Digitalisierung die Bedeutung des Netzwerks und die damit erforderliche Steigerung der Vernetzungsdichte. An Stelle von Zielvereinbarung und Kontrolle sieht er die Gestaltung optimaler Rahmenbedingungen für Kooperation.

Das Konzept einer hierarchisch steuernden Führung steht mit der digitalen Transformation vor seinem Offenbarungseid. An die Stelle rigider Weisungs- und Berichtsketten tritt zunehmend die Impulskraft moderierter, sich selbst organisierter Netzwerke.

Er vergleicht Führen im digitalen Umfeld mit dem Hüten von Katzen. Eigensinnig, unabhängig und gelegentlich kapriziös und damit anders als die gehorsamen und folgsamen Hunde, stellen Mitarbeiter*innen das ArbeitgeberArbeitnehmer-Verhältnis zunehmend auf den Kopf. Flexible Arbeits- und Kooperationsmodelle untergraben die Bindung gut qualifizierter, innovativer und kreativer Mitarbeiter*innen an das Unternehmen. Die Arbeit selbst, die Freude daran und die Entfaltungsmöglichkeiten der eigenen Potenziale gewinnen zentralen Stellenwert. Eine ergebnisorientierte Führung jenseits starrer

\begin{tabular}{|c|c|}
\hline 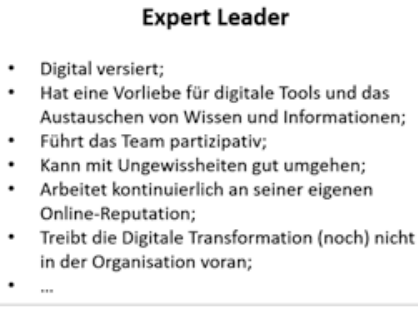 & \begin{tabular}{l}
\multicolumn{1}{c}{ Digital Leader } \\
- Zeigt alle Eigenschaften eines Expert Leaders; \\
- Treibt die Digitale Transformation in der Organisation \\
voran; \\
- Agiert als aktives Vorbild bei der Kulturentwicklung; \\
- Hat eine klare und attraktive Vision, die andere \\
inspiriert; \\
- Weiß, wie er andere bei der Digitalen Transformation \\
- $\quad$ Hat einbinden kann; \\
- $\quad$-..
\end{tabular} \\
\hline \begin{tabular}{l}
\multicolumn{1}{c}{ Conservative Leader } \\
- Verfolgt einen traditionellen Führungsstil; \\
- Vorhersagbarkeit gibt ihm Sicherheit; \\
- Noch nicht digital versiert; \\
- Bevorzugt die persönliche Kommunikation; \\
- Ist vorsichtig gegenüber der Digitalen \\
Transformation und neuen Arbeitsformen; \\
- Erforscht für sich noch die Wirkung einer \\
Digitalen Transformation; \\
- ...
\end{tabular} & $\begin{array}{l}\text { Change Leader } \\
\text { - Kennt die Relevanz der Digitalen Transformation für } \\
\text { das Geschäftsmodell und weiß um die Notwendigkeit } \\
\text { organisationaler Anpassungen; } \\
\text { - Nutzt selbst wenig digitale Tools, administriert aber } \\
\text { andere bei deren Nutzung; } \\
\text { - Eher traditionelle Führung; } \\
\text { - Experimentiert mit neuen Arbeitsweisen; } \\
\text { - Begrüßt den Wandel; } \\
\text { - Treibt die Digitale Transformation in der Organisation } \\
\text { mit voran. }\end{array}$ \\
\hline
\end{tabular}

Intensität des Transformationsmanagements

Abb. 4.4 Digital Leadership Matrix, Quelle: Capgemini Invent. (Eigene Übersetzung) 
Strukturen, verbunden mit Anerkennung und Wertschätzung der Mitarbeiter*innen, löst eine auf Kontrolle ausgerichtete Präsenzkultur ab.

Während die Digitalisierung traditionelle Arbeitskonventionen in Frage stellt, müssen sich Führungskräfte damit auseinandersetzen, wie sich den verändernden Rahmenbedingungen im Führungsverhalten Rechnung tragen lässt.

Buhse (2014), Experte und Vordenker für Digital Leadership, hat mit dem Akronym VOPA+ versucht eine Antwort darauf zu geben. Das VOPA+ Modell wurde gezielt als Antwort auf die Herausforderungen einer VUKA-Umwelt entwickelt. In seinem Zentrum steht die Vertrauenskultur. Diese dient als Basis, digitalen Veränderungsprozessen erfolgreich begegnen zu können. Die Anforderungen an die Führungskraft fasst das Modell in vier Dimensionen zusammen: „Vernetzung“, „Offenheit“, „Partizipation“ und „Agilität“.

Vernetzung: Es gilt Vernetzungen zwischen Menschen über verschiedene Kanäle und soziale Plattformen herzustellen. Führungskräfte sind dennoch gehalten, den direkten persönlichen Austausch zwischen den Mitarbeitenden zu ermöglichen.

Offenheit: Offenheit bedeutet, den gegenseitigen Wissensaustausch zu fördern und Informationen allgemein zugänglich zu machen. Eine Form des aktiven, kurzen Wissensaustauschs bildet beispielsweise ein Weekly Standup-Meeting. In diesem beantwortet jeder Mitarbeitende 3-4 zentrale Fragen wie: „Welche Aufgaben bearbeite ich während dieser Woche?“, „Welche Aufgaben/Projekte stehen zukünftig an?“”, „Wie hoch ist mein Auslastungsgrad?“" und „Wie geht es mir?““.

Partizipation: Partizipation bedeutet, kollektives Wissen anhand von konstruktiven, motivierenden und hierarchiefreien Formen des Austausches mit Einbezug möglichst vieler Personen zu nutzen - sei es in Form von Präsenzworkshops (z. B. Open Space) oder virtuellen Workshops über Onlineplattformen (z. B. der Cloud-Lösung Trello oder bank-/sparkasseninterne Plattformen). Dabei hat jede(r) Mitarbeitende die Gelegenheit, sich einzubringen.

Agilität: Wesentliches Merkmal von Agilität ist, sich schnell an ein sich permanent veränderndes Umfeld anpassen zu können und aus den gemachten Erfahrungen zu lernen. Agilität bezeichnet auch die Bereitschaft, Neues zu wagen sowie eigene Lösungsansätze und Handlungsoptionen zu entwickeln.

Vertrauen (entspricht dem +): Eine Vertrauenskultur zu schaffen heißt, in die fachlichen und sozialen Fähigkeiten der Mitarbeitenden zu vertrauen und ihnen die Möglichkeit zur Selbststeuerung und -verantwortung zu geben. 
Führungskräfte, die das VOPA+ Modell in ihrer Werthaltung verinnerlicht haben, richten ihre Alltagshandlungen danach aus. Sie sind in der Lage, in sich ständig wechselnden Kontexten die verschiedenen Führungsstile agil und flexibel anzuwenden. Das hilft ihnen im digitalen Zeitalter erfolgreich zu führen.

\section{Praxisbeispiel}

Teilweise ist für einzelne Führungskräfte nicht unmittelbar nachvollziehbar, worum es genau geht, wenn von einem Aufbau von digitalen Kompetenzen gesprochen wird. Grundsätzlich sind immer alle dafür, mehr digitales Know-how in die Bank/Sparkasse zu bringen. Wie dies aber konkret bei den Mitarbeiter*innen erfolgen soll, ist nicht immer transparent.

So musste, wie in einem Fall, dem Vorstand klar werden, dass sich die Wahrscheinlichkeit zum Erreichen der strategischen Ziele, wie z. B. „ein agiles Vorgehen zur Förderung von Innovationen etablieren“ oder „Digitale Angebote entwickeln und Produkte digitalisieren" dadurch erhöht, dass die Mitarbeiter*innen der Bank ihrerseits ihre digitalen Kompetenzen aufbauen. Dadurch lenken sie ihr Verhalten stärker in die gewünschte Richtung und zahlen damit mittelbar oder unmittelbar auf diese Ziele ein. Erst nach dieser Erkenntnis gab er die erforderlichen Projektschritte zur Entwicklung einer Kompetenzsystematik frei.

Auch in den Fachbereichen reicht es oft nicht aus, digitale Kompetenzen und Verhaltensanker zu definieren (siehe Abschn. 6.4). Häufig nimmt dies für die Anwendung eine zu hohe Abstraktionsebene ein. So bedarf es einer zusätzlichen Übersetzung, was dies in der Arbeitspraxis des Mitarbeiters/der Mitarbeiterin bedeutet. Beispielsweise wirkt sich dies folgendermaßen aus:

- Aufbau und Nutzung einer CRM (Customer-Relationship-Management) -Systematik.

- Einsatz eines Ticket-Systems/der Vorgangssteuerung zur Schaffung von Transparenz zu Arbeitsabläufen.

- Einbindung von Recherche-Ergebnissen im Internet in die eigene Arbeit.

- Nutzung von Foren, Suchmaschinen, Netzwerken und weiteren Plattformen zur Erweiterung des eigenen Wissens, des Wissens des Teams/der Bank/Sparkasse.

- Berücksichtigung von Vorgaben bei fallabschließenden Vorgängen.

- Bedachte und zielorientierte Nutzung der digitalen Anwendungen und Geräte in der Bank/Filiale/am Arbeitsplatz. 
- Einbindung von Spezialisten in den Beratungsprozess über Videokanäle.

- Unterstützung von Kolleg*innen per TeamViewer (oder ähnlicher Software) bei Problemlösungen.

- Aktives Einbringen in hausinterne Foren im Intranet statt reine Konsumentenhaltung.

- Adressatengerechte Kommunikation per Chat und Mail (Einhaltung der Netiquette).

- Beteiligung am Aufbau von Wissensdatenbanken im Team/der Abteilung/ der Bank/Sparkasse.

- Effizienter Einsatz arbeitsplatzbezogener Tools.

- Nutzung hausinterner Chats und Foren zum Austausch und zur Diskussion.

- Sicherer Umgang mit Mobile Devices (Mobile Phones, Tablets etc.) im Kundenkontakt.

Bereits heute und künftig noch viel mehr wird es notwendig sein, persönliche Bindung auch über unpersönliche, digitale Kanäle zu gewährleisten. Im Kundenverkehr ist dies in Banken und Sparkassen seit langem Normalität. In der Führung von Mitarbeiter*innen stellt dies jedoch nach wie vor ein großes Lernfeld dar, das uns zum nachfolgenden Führungsstil bringt.

\section{Fazit aus diesem Abschnitt}

Führungskräfte müssen sich selbst fit machen, um die Herausforderungen der digitalen Arbeit bewältigen zu können. Die Gestaltung optimaler Rahmenbedingungen für eine Kooperation der Mitarbeiter*innen innerhalb und außerhalb der Bank/ Sparkasse stellt eine weitere zentrale Erfolgskomponente für Partizipation und Agilität dar. Der Auf- und Ausbau digitaler Kompetenzen schafft mehr Optionen, um das Geschäftsmodell der Bank/Sparkasse weiterzuentwickeln.

\subsection{Distance Leadership/virtuelle Führung}

In der Banken- und Sparkassenwelt reißt der Trend zu immer größer werdenden Organisationseinheiten und Geschäftsgebieten nicht ab (vgl. Zeb 2018).

Dabei gibt es durchaus Erfahrungen aus der Praxis, dass sich kleinere Organisationseinheiten besser händeln lassen. 
Eines dieser Beispiele ist W. L. Gore. Das Unternehmen, das vor allem durch seine wasserdichte Bekleidungsmembran Gore-Tex bekannt ist, zählt nach wie vor zu den erfolgreichsten Unternehmen der Welt.

Es hat bei mehr als 10.000 Mitarbeitenden nur drei Hierarchie-Ebenen: Es gibt den/die (demokratisch gewählte/n) CEO, eine Handvoll Abteilungsleiter*innen und den ganzen Rest. Sämtliche Entscheidungen fallen in selbstverwalteten Teams mit acht bis zwölf Mitgliedern - ob über Neueinstellungen, die Bezahlung oder über die Art der bearbeiteten Projekte. Statt formeller Organisationsstrukturen gibt es informelle. An die Stelle von Vorgaben und Anweisungen treten kulturelle, auf Vertrauen basierende Abmachungen unter den Mitarbeiter*innen.

In Deutschland hat der Mittelständler neun Werke ausschließlich in Bayern. Jedes Werk bildet eine eigenständige Zelle von maximal 200 Mitarbeiter*innen. Der Vorteil dieser kleinen, eigenständigen Werke: höhere Effizienz. Denn schon bei mehr als 150 Mitarbeiter*innen kann es schwierig werden, mit allen Arbeitskolleg*innen schnell und unmittelbar zu kommunizieren und sich umeinander zu kümmern. Deshalb ist die Größe der einzelnen Werke begrenzt. Wächst ein Werk über 200 Personen im Schichtbetrieb hinaus, muss ein Neues her oder die Arbeit wird in ein anderes verschoben.

Die Nähe der Werke zueinander und die flache Hierarchie ermöglichen es den Mitarbeiter*innen, sich auch zwischen den Zellen schnell und direkt auszutauschen. Jeder kann mit jedem sprechen und jeder soll jedem behilflich sein. Barrieren durch vorgegebene hierarchische Strukturen werden vermieden.

Wachsende Organisationseinheiten, z. B. durch Fusionen, haben bei Finanzinstituten häufig zur Folge, dass sich das Geschäftsgebiet flächenmäßig ausweitet. Um nicht zu viele Hierarchieebenen einzuführen, tragen Führungskräfte dann oft die disziplinarische Verantwortung für mehrere Standorte.

Hinzu kommt, dass eine zunehmende Anzahl von Mitarbeiter*innen im Home-Office oder mobil ihre Arbeit verrichtet. Die Besonderheiten eines international ausgerichteten Finanzunternehmens mit den damit verbundenen kulturellen und sprachlichen Herausforderungen kämen noch hinzu, bleiben an dieser Stelle aber außen vor, um den Rahmen nicht zu weit auszudehnen.

Alle diese Rahmenbedingungen führen dazu, dass räumliche Distanzen entstehen, die eine starke zeitliche Präsenz der Führungskräfte bei ihren Teams und Mitarbeiter*innen vor Ort nicht mehr im gewünschten Maße möglich machen und damit die direkte Kommunikation eingeschränkt ist. 
Das ist nicht wirklich neu. Heute stehen jedoch vielfältige technologische Möglichkeiten zur Verfügung, mit deren Unterstützung Nähe zu den Mitarbeiter*innen auf unterschiedliche Art und Weise hergestellt werden kann.

Kommunikationskanäle, wie z. B. Videokonferenzen, Cloud-Working und Net Meeting bieten gute Gelegenheiten, Informationen auszutauschen und Teammitglieder in Austausch miteinander zu bringen.

Wald (2014) kommt daher folgerichtig zu der folgenden Definition:

Erfolgt aufgrund des Mangels an direkten Kontakten die wechselseitige Einflussnahme zwischen Führungskräften und Geführten hauptsächlich mit Hilfe neuer Informations- und Kommunikationsmittel (IuK) beziehungsweise mittels sozialer Medien, so wird dies hier grundsätzlich als virtuelle Führung verstanden.

Was aber ist erforderlich, um Teamarbeit im virtuellen Raum bzw. auf Distanz wirklich effizient zu gestalten?

Wie gut die einzelnen Häuser auf die dabei erforderlichen Rahmenbedingungen eingestellt sind, zeigte sich durch die Extremsituation im Frühjahr 2020 als zahlreiche Mitarbeiter*innen aufgrund der Präventiv- oder Quarantänemaßnahmen zum Covid-19 (Coronavirus) ihre Arbeit von zu Hause aus verrichten mussten.

Bereits die Sicherstellung einer effektiven Kommunikation stellt im virtuellen Raum eine nicht zu unterschätzende Herausforderung dar. Einfaches lässt sich per Mail, Textnachricht oder internem Messagingsystem klären. Dringendes und Klärungsbedürftiges via Telefon oder Live-Chat und Komplexes am besten mit Hilfe einer Videokonferenz oder dann doch wieder persönlich von Angesicht zu Angesicht.

In der Anfangsphase eines virtuellen Teams bedarf es daher einer hohen Unmittelbarkeit von Feedback, d. h. einer möglichst synchronen Kommunikation mit geringen Verzögerungen. Hierzu tragen z. B. Face-to-Face-Meeting, Telefon-, Videokonferenz, aber auch gute Nachbereitungsmöglichkeiten, wie z. B. Net Meeting-Aufzeichnungen bei. Zusätzlich sind viele symbolische Informationen zur reinen Nachricht, wie z. B. mimische Hinweise oder der Klang der Stimme hilfreich, um die Sozialisation und Teambildung zu fördern.

Remdisch (2005) hebt die Vorteile und Nachteile virtueller Teams hervor. Tab. 4.4 stellt diese gegenüber.

Sie betont insbesondere das Dilemma, in einer virtuellen Welt Vertrauen aufzubauen. Vertrauen entsteht durch Face-to-Face-Interaktionen. Es entsteht mit der Zeit und je besser man andere kennt. Die virtuelle Welt jedoch beruht auf einem entgegengesetzten Prinzip: Schnelle Kontakte und eine Zusammenarbeit mit weitgehend Unbekannten. 
Tab. 4.4 Vor- und Nachteile virtueller Teams

\begin{tabular}{l|l}
\hline Vorteile & Nachteile \\
\hline $\begin{array}{l}\text { Flexible Einbindung von Expert*innen } \\
\text { verschiedener Standorte }\end{array}$ & $\begin{array}{l}\text { Geringere Identifikation mit der } \\
\text { Organisation/dem Team }\end{array}$ \\
\hline $\begin{array}{l}\text { Teamzusammensetzung orientiert sich nach } \\
\text { Fähigkeiten, nicht nach Verfügbarkeit }\end{array}$ & $\begin{array}{l}\text { Unsicherheit bzgl. Aufgaben, Rollen, } \\
\text { Verantwortlichkeiten, Prioritäten }\end{array}$ \\
\hline $\begin{array}{l}\text { Hoher Grad an Selbstorganisation der } \\
\text { Mitarbeiter*innen }\end{array}$ & Aufbau von Vertrauen ist schwierig \\
\hline $\begin{array}{l}\text { Reduktion von Reisekosten für } \\
\text { Teammitglieder }\end{array}$ & Gefühl der Isolation \\
\hline $\begin{array}{l}\text { Optimierung der Zeitressource (reduzierte } \\
\text { Reisezeiten, ggf. 24h-Arbeit) }\end{array}$ & $\begin{array}{l}\text { Abhängigkeit von Informations- und } \\
\text { Kommunikations-Technologie }\end{array}$ \\
\hline $\begin{array}{l}\text { Optimierte Informationsversorgung } \\
\text { Erhöhter Organisationsaufwand für } \\
\text { lokale Treffen }\end{array}$ \\
\hline & Schwierigkeit des Leistungs-Feedbacks \\
\hline & $\begin{array}{l}\text { Missverständnisse und Konflikte } \\
\text { entstehen schneller }\end{array}$ \\
\hline
\end{tabular}

Eigene Darstellung in Anlehnung an Remdisch (2005)

Das Dilemma liegt darin, dass die Schwierigkeit, Vertrauen aufzubauen, mit einem zunehmenden Grad an Virtualität steigt. Gleichzeitig setzt das Arbeiten im virtuellen Team starkes Vertrauen in Personen voraus, die man nur virtuell kennt.

Als eine zentrale Aufgabe einer virtuellen Führungskraft benennt sie daher den Aufbau von Vertrauen im Team. Gelingen kann dies durch die folgenden Aufgaben, die wiederum nicht wesentlich von denen in realen Teams abweichen:

- Aktiv steuern, Informationsaustausch organisieren, Interaktion fördern (nicht nur aufgabenbezogene, sondern auch soziale Kommunikation);

- Eigenschaften/Qualifikationen jedes einzelnen Teammitglieds, die zum Teamerfolg beitragen sollen, benennen;

- Beiträge einzelner zum Teamerfolg hervorheben;

- Anzeichen für Vertrauensbrüche nachgehen und Vertrauen wiederherstellen;

- Misserfolge konstruktiv kommunizieren/das Lernpotenzial vermitteln;

- Aufgabenkonflikte nutzen, um kreative Lösungen zu finden (statt Personenkonflikte daraus entstehen zu lassen).

Häufig herrscht die Meinung vor, dass im virtuellen Raum völlige Freiheit für alle Beteiligten existiert. Das Gegenteil ist der Fall. Von allen Beteiligten sind mit hoher Disziplin eher eine größere Anzahl von Regeln einzuhalten als bei der $\mathrm{Zu}-$ sammenarbeit realer Teams. 


\section{Fragen}

Die Klärung folgender Fragen können die Arbeit (nicht nur) im virtuellen Team unterstützen:

- Ob, wann und wie regelmäßig sollen Treffen stattfinden?

- Wie werden Entscheidungen getroffen?

- Welche Erwartungen haben die Teammitglieder aneinander?

- Wie wird Feedback gegeben (im Team und vom Team-Leader)?

- Wie wird Kritik formuliert und wie wird damit umgegangen?

- Wie wird mit der Nichtbeachtung von Absprachen umgegangen?

- Wie werden E-Mails gehandhabt (wann abgerufen, wann beantwortet)?

- Wie kann man im Team Unterstützung einholen?

- Wie wird mit Erfolgen/,Spaß“ umgegangen?

- Wie wird mit Vertraulichkeit umgegangen (im Team/nach außen)?

Die Führungskraft auf Distanz muss Arbeitsaufgaben präzise strukturieren, eine gute Einarbeitung gewährleisten und dafür sorgen, dass Ziele, Aufgaben und Verantwortlichkeiten klar sind. Denn im virtuellen Raum werden Missverständnisse bei Aufgaben und Entscheidungen, Schwierigkeiten bei der Aufgabenerreichung, fehlerhafte Prozesse, etc. oftmals erst viel später erkannt als bei realen Gruppen.

Virtuelle Teams effektiv einzusetzen, ist ein diffiziles Unterfangen: Die Personalberatung Rochus Mummert (2013) stellt fest, dass rund $75 \%$ der virtuellen Teams aufgrund der hohen Anforderungen an Teammitglieder und vor allem an Führungskräfte scheitern. Von der Führung traditioneller Teams bekannte Führungsherausforderungen potenzieren sich für Teamleitungen virtueller Teams.

Akin und Rumpf (2013) betonen, dass vor der virtuellen Zusammenarbeit meist keine vorbereitenden Trainingsmaßnahmen stattfinden, weder für Führungskräfte noch für Teammitglieder. Und dies, obwohl es einer immensen Vorbereitung von Führungskräften und Teammitgliedern auf die Arbeit in virtuellen Teams bedarf.

Wie sollte erfolgreiches Führungsverhalten in virtuellen Teams ausgestaltet sein?

Fajen (2017) nennt:

- Regelmäßige elektronische Kommunikation (bilateral als auch im gesamten Team) initiiert durch die Führungskraft;

- Einsatz reichhaltiger Kommunikations- und Informationsmedien; 
- Teammitgliedern die notwendige Infrastruktur für die elektronische Kommunikation zur Verfügung stellen;

- Die Führungskraft sollte Sprache bewusst einsetzen und sich rückversichern, ob Informationen wie beabsichtigt angekommen sind;

- Organisation gelegentlicher Face-to-Face Treffen;

- Die Führungskraft sollte über den genauen Standort und die Rahmenbedingungen der Arbeit ihrer Teammitglieder informiert sein;

- Informationsfilterung und gleichzeitige Informationsdistribution;

- Die Führungskraft sollte erreichbar sein und virtuelle Präsenz zeigen (z. B. durch eine Politik der offenen virtuellen Tür und ein schnelles Reagieren auf Anfragen);

- Teammitgliedern klar strukturierte Aufgaben und regelmäßiges Feedback geben, um selbstständige Arbeit zu ermöglichen.

Grote et al. (2004) verweisen in diesem Zusammenhang auf das Sensing als eine der wesentlichen Führungsaktivitäten virtueller Teamleiter*innen. Sensing fokussiert auf die visuellen und die auditiven Sinnesmodalitäten und bedeutet, dass Leiter*innen virtueller Teams diese Sinnesmodalitäten für die im virtuellen Raum agierenden Menschen schärfen müssen. Dies insbesondere dafür, wie sie denken, fühlen und handeln, aber auch dafür, wie sie selbst über Informations- und Kommunikationsmedien wirken.

Abb. 4.5 verdeutlicht, dass virtuelle Führung keinen eigenständigen Führungsstil darstellt, sondern vielmehr als integrativer Ansatz diverse Führungsstile und Verhaltensansätze in sich vereint.

\section{Praxisbeispiel}

In der Praxis von Banken und Sparkassen erfährt virtuelle Führung eine große Interpretations-Bandbreite. Einige Institute sammeln gerade erst Erfahrung mit mobilen oder Home-Office Arbeitsplätzen, der sogenannten alternierenden Telearbeit. Für andere Institute ist dies bereits regelmäßiger Bestandteil ihrer Führungspraxis.

Ein Aspekt zeigt sich bei der Führung auf Distanz jedoch durchgängig: Damit Vertrauen auf Distanz aufgebaut werden kann, sind regelmäßige PräsenzTreffen unabdingbar. Technologie kann Distanzen zwecks Kommunikation und zum Austausch untereinander wunderbar überbrücken. Wenn es aber menscheln soll, bedarf es auch der Menschen hierzu. So berichtet eine Bereichsleiterin einer Bank in einem Workshop darüber, dass sie in Anlehnung an die Systematiken und Arbeitsmethoden der agilen Arbeit regelmäßige Treffen aller Teammitglieder organisiert, um persönliche Beziehungen untereinander zu fördern. 


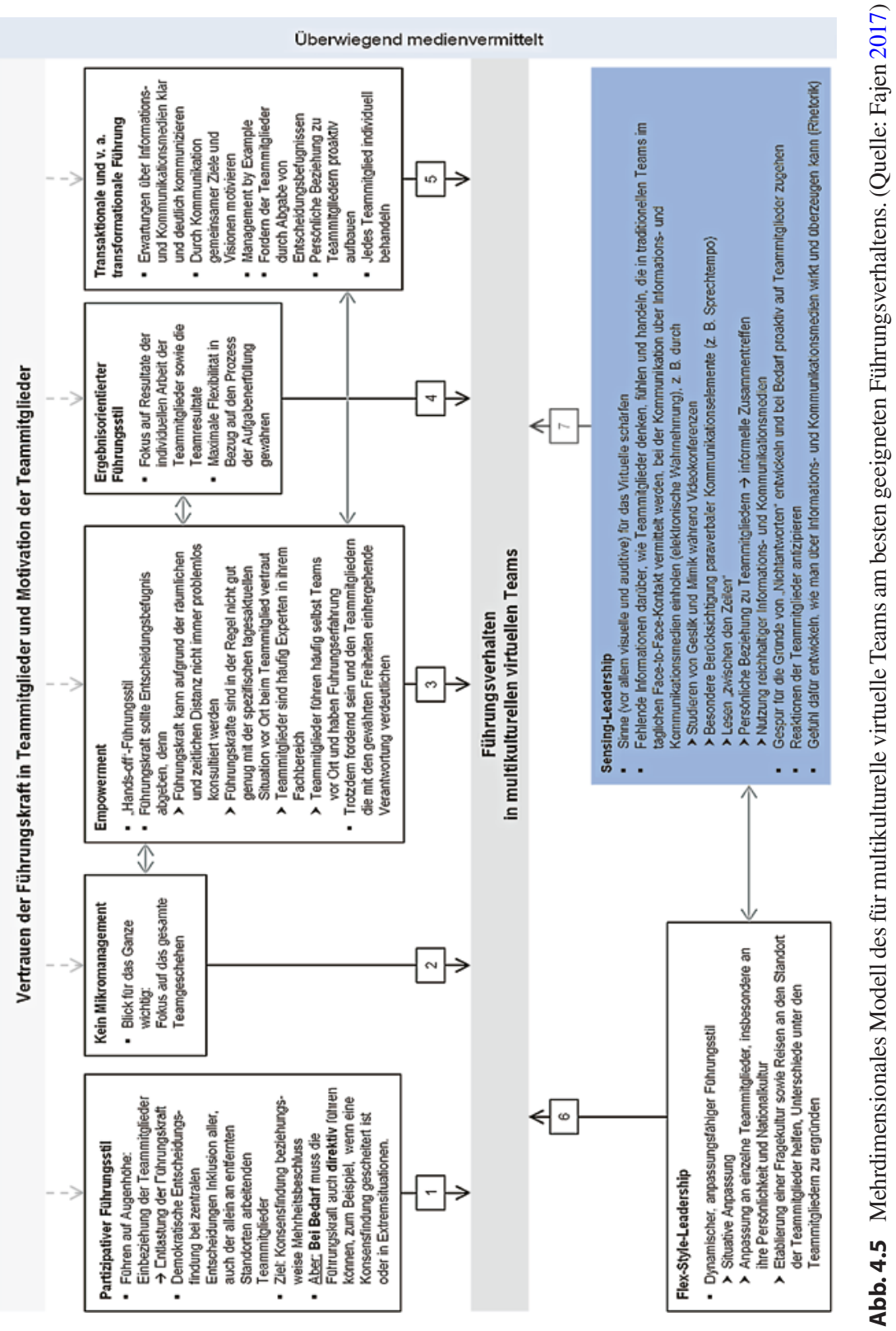


Während der räumlichen Trennung wird der persönliche Austausch durch eine Chatfunktion auf den Arbeitsgeräten der Mitarbeiter*innen unterstützt. Dort werden in erster Linie persönliche, nichtfachliche Themen ausgetauscht. Den fachlichen, regelmäßigen Austausch versucht sie neben dem gängigen Mailverkehr einmal pro Woche zum Wochenstart über kurze, halbstündige Video- oder Telefonkonferenzen mit allen, die gerade Zeit und die Möglichkeit hierzu haben, sicherzustellen. Das Prinzip Selbstverantwortung setzt sie als hohes Gut bei allen Mitarbeiter*innen an. Im Mittelpunkt des Führens stehen die eigenverantwortlichen Aktivitäten der Mitarbeiter*innen, deren Umsetzung der Aufgaben und die daraus resultierenden Umsetzungsergebnisse. Am Ende der Woche ruft sie bei jedem Mitarbeiter/jeder Mitarbeiterin persönlich an, um das persönliche Befinden zu erkunden und kurz die Woche Revue passieren zu lassen. Zu viel des Guten? Vertrauen braucht eben eine gewisse Nähe, auch oder vor allem in der virtuellen Welt.

\section{Fazit aus diesem Abschnitt}

Die Aufgaben von Führen in virtuellen Teams/bei der Führung auf Distanz unterscheiden sich augenscheinlich nicht von denen bei der Führung von Präsenzteams. Um Nähe über Distanz zu gewährleisten und Vertrauen auf Distanz aufzubauen bzw. aufrecht zu erhalten, bedarf es einer Kombination aus einer effizienten Nutzung technischer Möglichkeiten und einem regelmäßigen Austausch auch auf der persönlichen Ebene in Präsenztreffen. Führung sollte den persönlichen Kontakt zu Beginn der Arbeit auf Distanz intensiver gestalten und dann im weiteren Verlauf je nach Komplexität des dahinterliegenden Sachverhaltes angemessen ausrichten.

\subsection{Agile Führung/Agiles Coaching}

Ist es eine vorübergehende Modeerscheinung oder doch eine zwingende Notwendigkeit, sich mit Agilität und den damit verbundenen Organisations- und Arbeitsstrukturen und veränderten Führungsaufgaben in der Bank oder Sparkasse auseinandersetzen zu müssen? Und was meint Agilität überhaupt im Unternehmenskontext von Banken und Sparkassen?

Dass Agilität an sich nicht neu ist, ist unbestritten. Bereits vor über vierzig Jahren hat es sich in der Softwareentwicklung durchgesetzt, schrittweise vorzugehen und damit eine zuverlässige Basis für Projektpläne zu schaffen. Dies ist heute in diesem Bereich eine gängige Praxis. 
2001 entwickelten einige Verfechter dieser Methodik das „Agile Manifest der Softwareentwicklung“. Damit begann die Verbreitung des Begriffs ,agil“ als Bezeichnung für eine neue Vorgehensweise und Organisation im Gegensatz zur traditionellen.

Agiles Vorgehen zeichnet sich aus durch:

- Vorgehen: Kleine Schritte mit kurzfristigem zeitlichen Rahmen, die aufeinander aufbauende, bereits benutzbare Teilergebnisse liefern;

- Kontrolle: Kontinuierliche Überprüfung der Teilergebnisse und der Arbeitsweise unter regelmäßiger Einbindung der Nutzer;

- Planung: Kontrolle als Basis für die Adaption der Planung der nächsten Schritte.

Dieses Vorgehen funktioniert umso besser, je mehr die folgenden Voraussetzungen erfüllt sind:

- Flexibilität: Bereitschaft für ein der Situation angepasstes Vorgehen statt strikter Einhaltung von Prozessen;

- Offenheit: Bereitwilliges Aufnehmen neuer Kundenwünsche und Einsichten statt konsequenter Einhaltung längerfristiger Pläne;

- Zusammenarbeit: Weitgehend autonome, sich selbst organisierende, funktionsübergreifend zusammengesetzte und von teamexternen Personen unabhängige Teams;

- Form: Vorrang der persönlichen, spontanen Kommunikation vor Schriftlichkeit.

Der vermeintliche Siegeszug dieser Vorgehensweise bei der Softwareentwicklung hat zu einer erheblichen Verbreitung von ,agil“ geführt und zum Bestreben, auch Bereiche außerhalb der Softwareentwicklung agiler zu gestalten.

Korn (2016) gibt zu bedenken:

All dies bedeutet in vielen Unternehmen eine erhebliche Veränderung gegenüber etablierten „Spielregeln“ und gipfelt oft in Idealvorstellungen einer insgesamt ,,agilen Organisation" die sich von unten nach oben ohne Manager und ohne Anweisung und Kontrolle gestaltet. 
Dies führt nicht selten dazu, dass Organisationen mit der Einführung agiler Strukturen überfordert sind und an der Umsetzung in der Praxis scheitern.

\section{Praxisbeispiel}

Banken und Sparkassen gehen verstärkt dazu über, Schlüsselfunktionen für die Entwicklung und Einführung von Innovationen im Sinne eines CDO (Chief Digital Officers) einzurichten. Dies ist grundsätzlich sinnvoll, rückt die Thematik damit näher an die Vorstandsebene. Da diese Funktionen aber oft als Querschnittsfunktionen daran gemessen werden, wie schnell sie Neuerungen umsetzen, stoßen sie häufig auf Widerstände in den klassischen Linienfunktionen.

Die Fachabteilungen und Bereiche verteidigen häufig ihre, nicht selten in Eigenentwicklung, aufgebauten Systeme und Vorgehensweisen als einzig funktionierende Lösung. Greift z. B., wie in einer Praxisbank erfolgt, ein Innovationsverantwortlicher in diese Konzepte durch Prozessveränderungen ein, fühlen sich die Fachverantwortlichen in ihrer Freiheit beschnitten und in ihrer zurückliegenden Arbeit nicht ausreichend gewürdigt. Hilfreich sind daher eine hohe Transparenz zur Sinnhaftigkeit möglicher Neuerungen für die betroffene Abteilung/den betroffenen Bereich sowie eine frühzeitige Einbindung der Betroffenen in den Veränderungsprozess.

Alberts und Hayes (2009) schlagen vor, ein abgewandeltes Verständnis von agil zu verfolgen, das einer eher hybriden Vorgehensweise entspricht und sowohl ein inkrementell-adaptives Vorgehen als auch ein stark planbestimmtes und auf strikt einzuhaltende Prozesse abgestütztes Vorgehen ermöglicht.

Nach ihren Vorstellungen ist eine Organisation dann ,,agil“, wenn die sie bildenden Menschen die folgenden Fähigkeiten und Eigenschaften mitbringen:

1. Robustheit: die Fähigkeit, aufgaben-, situations- und bedingungsübergreifend effektiv zu bleiben

2. Belastbarkeit: die Fähigkeit, sich von Unglücksfällen, Schäden oder einer destabilisierenden Störung der Umgebung zu erholen oder sich darauf einzustellen

3. Reaktionsfähigkeit: die Fähigkeit, auf eine Veränderung der Umgebung rechtzeitig zu reagieren

4. Flexibilität: die Fähigkeit, mehrere Lösungsmöglichkeiten einzusetzen und nahtlos von einer zur anderen überzugehen 
5. Innovationsfähigkeit: die Fähigkeit, neue Dinge zu tun und alte Dinge auf eine neue Art und Weise zu tun

6. Anpassungsfähigkeit: die Fähigkeit, Arbeitsprozesse zu ändern und die Organisation zu ändern.

Nur mal rein theoretisch: Wenn die Mitarbeiter*innen von Banken und Sparkassen diese Fähigkeiten mitbringen, braucht es dann überhaupt noch Führungskräfte?

Korn (2016) behauptet, erfolgreiche Führung war schon immer agil. Diese These untermauert er dadurch, dass er Führung auf vertrautem, als stabil und sicher eingeschätztem Boden als mittelfristig entbehrlich hält. Er sieht Führung dann als nützlich, wenn sie die Entwicklung der oben genannten Fähigkeiten und deren Einsatz durch die Mitarbeiter*innen unterstützt und wenn ihr Ausmaß und ihre Art zur jeweiligen Situation passt.

Um sich Klarheit darüber zu verschaffen, welche Arten von Führung geeignet sind, mit Unsicherheit und Unplanbarkeit umzugehen, formuliert er folgende Fragen:

Braucht es auf unsicherem Boden vor allem charismatische und durchsetzungsstarke Führungspersonen mit einem ausgezeichneten Gespür für den richtigen Weg in hochkomplexen Situationen? Oder brauchen wir die freie Kooperation vieler entscheidungskompetenter Mitarbeiter*innen?

Braucht es trotz allem möglichst klare Strukturen und Verantwortlichkeiten, um die Komplexität nicht noch zu erhöhen?

Wie strikt darf und muss Führung sein?

Ist selbstorganisierte Teamarbeit eine unrealistische Idealisierung?

Oestereich und Schröder (2017) plädieren für die Selbstorganisation und eine damit verbundene kollegiale Führung. Als Merkmale hierfür führen sie auf:

- Ein hierarchisch organisiertes, soziales System, bei dem die Verantwortung dynamisch und situativ an die jeweils passenden Personen verteilt wird;

- Schnelle und flexible Entscheidungen;

- Interne Freiräume und Möglichkeiten;

- Einfache Führungs- und Organisationswerkzeuge, die von jedem Mitarbeiter/ jeder Mitarbeiterin einfach angewendet werden können;

- Führung als selbstverständliche Ergänzung der wertschöpfenden Arbeit und nicht nur auf exklusive Führungskräfte beschränkt; 
- Berücksichtigung beider Aspekte: Hohe Komplexität und Dynamik sowie effiziente, leistungsfähige Prozesse;

- Klare, sichere, belastbare, aber flexible Unternehmensstrukturen. Möglichkeit der Skalierung und Aufrechterhaltung von Innovation;

- Der Einzelne kann in seinem Rahmen eigenverantwortlich Ideen umsetzen. Hierfür hat jeder die Chance übergreifende Ressourcen zu erhalten;

- Nutzung der Unterschiedlichkeit und Vielfalt der Menschen, statt Gleichmacherei;

- Pragmatische Anwendung sozio- und holokratischer Grundprinzipien. Förderung wichtiger sozialer Werte und Haltungen;

- Viele Ideen und Praxisbeispiele zeigen, wie es funktionieren kann. Praktiken werden ergänzt durch passende Theorien und Denkmodelle;

- Zweck: Erzeugung von Kundennutzen, so profitabel, dass das Unternehmen für Inhaber*innen und Mitarbeiter*innen sinnvoll ist.

Ihre Organisationsarchitektur ist von kollegialen Kreisen geprägt: Geschäftskreise (Kreis der direkten Wertschöpfung, wie Privatkunden, Firmenkunden, Digitale Kunden etc.), Unterstützungskreise (Marketing, Personalwesen, interne IT etc.) und Koordinationskreise (Kreise für übergreifende Führungs-, Entscheidungs- und Koordinationsarbeit, wie z. B. Strategiekreis etc.).

Im Rahmen dieser Architektur und Systematik wird Führung über Prozesse und Werkzeuge anstelle von personenzentrierter Verantwortung abgebildet. Abb. 4.6 zeigt ein Musterbeispiel eines solchen Instruments, den Führungsmonitor.

Pull statt Push. Der Führungsmonitor setzt auf das Prinzip, dass sich jede(r) Einzelne einer Aufgabe verantwortungsbewusst annimmt. Das Ergebnis der Arbeit wird allen Beteiligten zur Reflexion bereitgestellt. Dies fördert gleichzeitig das Lernen der Organisation.

Auch Slogar (2018) postuliert in einer agilen Organisation von einer hierarchischen Führungsstruktur abzusehen. Stattdessen setzt auch er auf ein professionelles, verantwortungsbewusstes und distribuiertes Verständnis von Führung im Sinne einer Selbstorganisation und Selbstverantwortung der Mitarbeiter*innen.

Wolf (2003) wiederum sieht das Prinzip Selbstverantwortung kritisch, wenn er anmerkt:

Einerseits gehören nunmehr Selbstkoordination und kreative Problemlösung zum offiziellen Aufgabenkanon der Gruppe, andererseits fehlen Zeit und Personal, um diese Aufgaben angemessen erfüllen zu können. 


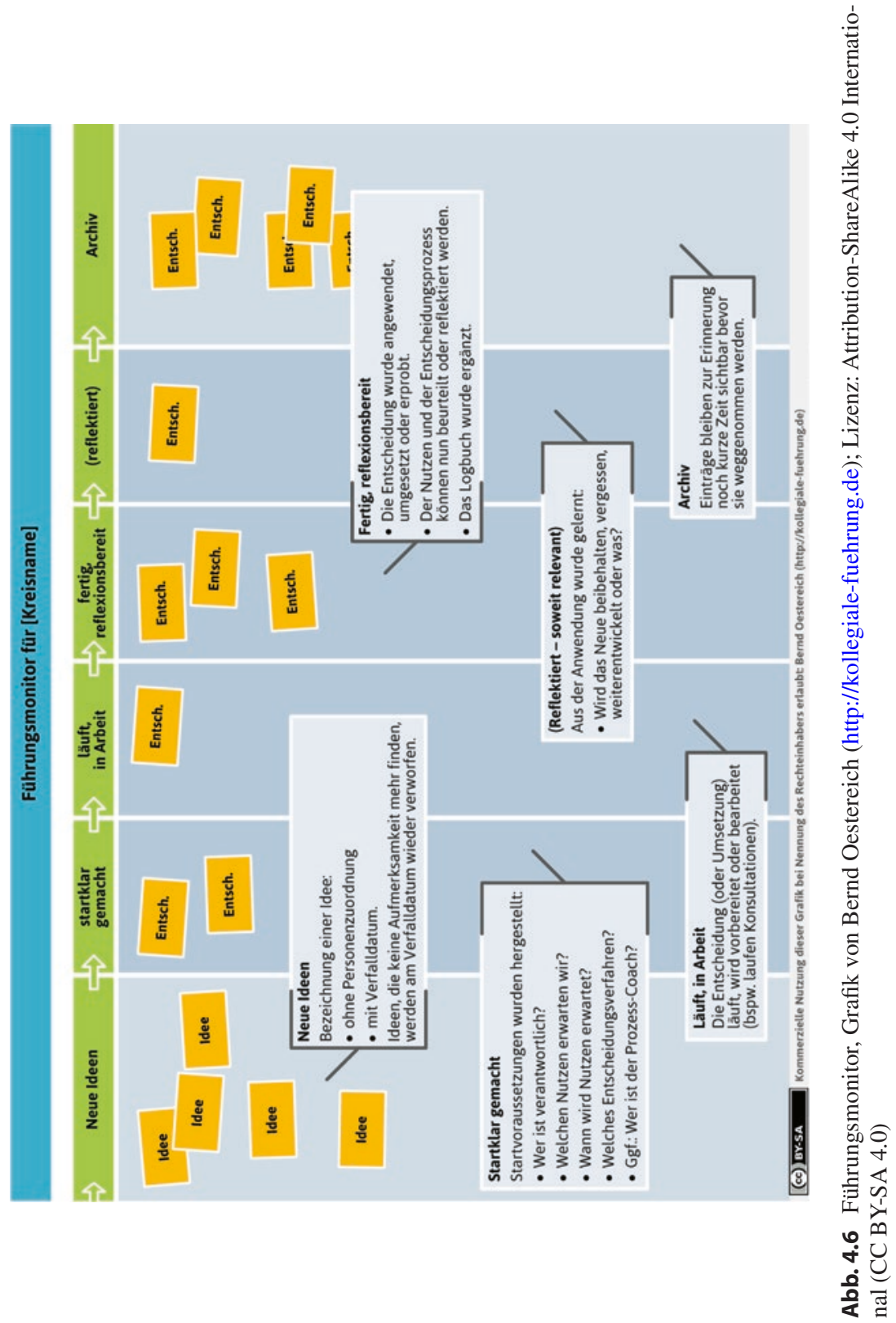


So ist es wiederum Korn (2016), der auf die Herausforderungen der Selbstorganisation z. B. in Matrixorganisationen hinweist. Innerhalb dieser werden Teams sehr oft aus Mitgliedern diverser Organisationslinien zusammengesetzt oder aus Personen, die jeweils mehreren Chefs/Chefinnen unterstellt sind (disziplinarisch einer Linienführungskraft, fachlich einer Marktsegmentleitung).

Bei der Matrixorganisation werden die nach Funktionen gegliederten vertikalen Linien von projekt- oder produktorientierten horizontalen Linien überlagert. Es entstehen dadurch Schnittstellen, welche von Mitarbeitenden oder von Teams besetzt werden können.

Mit der Matrixorganisation sollen die Vorteile aus Funktional- und Spartenorganisation kombiniert werden, indem das Unternehmen nach Funktionen und nach Sparten (bestimmten Objekten) untergliedert wird. Dadurch entsteht eine flexible Organisation, welche durch ein hohes Expertenwissen gekennzeichnet ist (vgl. Gabler Wirtschaftslexikon 2019).

Es liegt dann letzten Endes am Team oder gar am einzelnen Teammitglied selbst, die konkurrierenden Interessen und Vorgaben der verschiedenen ihm übergeordneten Organisationslinien unter einen Hut zu bringen. Dies scheitert regelmäßig daran, dass dem Team oder der Person in der Regel die Mittel und Entscheidungskompetenzen zur selbstständigen Gestaltung eines solchen Interessenausgleichs fehlen. In anderen Fällen sind die Kompetenzen zwar vorhanden, werden aber aus Angst vor Fehlern nicht wahrgenommen. Oder das Management greift trotz einer klaren Regelung zur Selbstverantwortung der Mitarbeiter*innen in der Matrix, im Sinne eines Mikromanagements, regelmäßig in die Entscheidungshoheit der operativen Ebene ein und hebelt damit die Matrix aus.

Geht man aber nicht von einer Agilität der kompletten Organisation aus, wie bei den Ausführungen zur Ambidextrie in Abschn. 3.3 angenommen, so hat Führung durchaus ihre Berechtigung. So z. B. im Kontext der Projektagilität oder wenn das eigene Team agiler aufgestellt werden soll (Freyth und Baltes 2017).

Schiefer und Nitsche (2019) geben einen umfassenden Überblick, wie sich die Rolle der Führungskraft in agilen Organisationen gestaltet. Als wesentlich stellen sie heraus:

- Rahmenbedingungen für agiles Arbeiten schaffen = unterstützende, steuernde und gestaltende Funktion (Hofert 2017);

- Hindernisse aus dem Weg räumen, erforderliche Ressourcen zur Verfügung stellen und Mitarbeiter*innen unterstützen, falls Hilfestellung erforderlich ist (Hofert 2017); 
- Einen offenen, hierarchieübergreifenden Dialog fördern, Transparenz und Vertrauen schaffen sowie eine konstruktive Feedback- und Fehlerkultur formen, die Lernen, Kritik, Veränderung und Eigenständigkeit ermöglicht (Häusling et al. 2017);

- Für eine effiziente Partizipation und Engagement jeder einzelnen Person sorgen und einen offenen Wissensaustausch fördern (Fox 2017);

- Ein übergreifendes Denken der Mitarbeiter*innen unterstützen, das konträr zum klassischen Silo-Denken die Wertschöpfung des Unternehmens im Gesamten in den Vordergrund stellt und nicht die Leistung eines Einzelnen (Lasnia und Nowotny 2018);

- Die Eigenständigkeit der Mitarbeiter*innen durch konsequentes Delegieren von Entscheidungen und Verantwortungen sowie dem Zuspruch bei der Entwicklung eigener Lösungsansätze fördern und fordern (Scheller 2017);

- Das entstehende Vertrauensverhältnis stetig überprüfen, um Vertrauensbrüche zu reflektieren (Geschwill und Nieswandt 2016);

- Für Produktivität und Ergebnisorientierung im Team sorgen. Der Fokus liegt auf der Leistung der Mitarbeiter*innen sowie auf einer maximalen Wertschöpfung. Dies stellt die Mitarbeiter*innen mit ihren Bedürfnissen und Kompetenzen stärker in den Mittelpunkt (Geschwill und Nieswandt 2016).

Führung übernimmt dabei immer mehr die Rolle eines Agilen Coachs.

Schiefer und Nitsche benennen jedoch auch Hindernisse von Seiten der Führungskräfte...

- Selbstwahrnehmung der Führungskräfte: Sie nehmen sich selbst agiler wahr, als ihre Mitarbeiter*innen sie erleben (Geschwill und Nieswandt 2016);

- Machtausübung seitens der Führungskraft: Im Rahmen agiler Personalführung können sie weniger Macht auf ihre Mitarbeiter*innen ausüben, da ihre Kontrollfunktion abnimmt und sie weniger Anweisungen erteilen können (Lasnia und Nowotny 2018). Das Machtmotiv ist bei vielen Führungskräften allerdings tendenziell eher stark ausgeprägt, da sie dadurch dem Streben nach Selbstbehauptung, Anerkennung, Kontrolle und Status nachgehen (Hofert 2017);

- Horten von Wissen: Im Rahmen des agilen Führungsverständnisses wird Wissen offen geteilt. Die Mitarbeiter*innen der Fachbereiche sind die neuen Expert*innen. Es gelingt Führungskräften nicht mehr, Informationen für sich zu behalten, um eine Expertenstellung oder Machtposition zu halten und sich dadurch unentbehrlich zu machen (Appelo 2010). 
...und von Seiten der Mitarbeiter*innen:

- Übernahme von Verantwortung: Manche Mitarbeiter*innen möchten aufgrund mangelnder Motivation oder Qualifikation nicht mehr Verantwortung übernehmen. Dies blockiert ihre Veränderungsbereitschaft (Geschwill und Nieswandt 2016);

- Überforderung und Unsicherheit: Mitarbeiter*innen können sich in einem volatilen Geschäftsumfeld unwohl fühlen und eine ständige Veränderung sowie die geforderte Flexibilität als Stressfaktoren erleben (Appelo 2010). Das kann Angst auslösen, die im Extremfall in der inneren oder in der tatsächlichen Kündigung endet (Fox 2017);

- Teamdynamik: Besteht ein Team aus Mitarbeiter*innen, die zusätzliche Verantwortung scheuen und sich gegen die Veränderung sträuben sowie einer Führungskraft, die an alten Führungsmustern hängt und nicht die Notwendigkeit zur Weiterentwicklung erkennt, existieren denkbar schlechte Bedingungen für agile Führung.

\section{Praxisbeispiel}

Um ihre Führungskräfte auf die Anforderungen der Agilität in der Bank vorzubereiten, hat ein Haus begleitend zu diversen Trainingsmaßnahmen ein Booklet herausgegeben. Dieses gibt neben den Erläuterungen zur Agilität, Hinweise zu den Auswirkungen für das Führungsverständnis und praktische Tipps zur Herangehensweise an veränderte Arbeitsbedingungen.

Es gibt den Führungskräften der Bank Impulse zu folgenden Fragestellungen/Aspekten:

- Warum beschäftigen wir uns mit dem Thema Agilität?

- Was genau ist Agilität auf der Persönlichkeitsebene, Kundenebene, Teamebene und Prozessebene?

- Was ist der Kern des agilen Arbeitens in der Bank (Werte, Prinzipien, Framework, Methoden und Techniken)?

- Standpunkt der Bank zur Agilität im Abgleich mit dem aktuellen Führungsverständnis (Merkmale der Bank, agile Merkmale, darauf einzahlende Führungskompetenz).

- Wo ist der Einsatz agiler Methoden in der Bank sinnvoll?

- Agile Führung und die Bedeutung für die Führungskräfte (Rolle, Kompetenzen, Herausforderungen beim Einführen von agilen Methoden). 
- Wie funktioniert die Umsetzung (mögliche Herangehensweise an agile Projekte)?

- Abgerundet wird der Handlungsleitfaden durch eine kleine agile Toolbox für den Praxisstart.

\section{Fazit aus diesem Abschnitt}

Immer mehr Banken und Sparkassen streben danach Teilbereiche ihres Hauses agiler auszurichten. Dabei macht eine hybride Vorgehensweise Sinn, d. h. es werden lediglich die Teilbereiche neugestaltet, die sich mit Innovationen oder einer iterativen, prozessualen Arbeitsweise (z. B. in der IT) auseinandersetzen. Der Selbstverantwortung aller Beteiligten kommt eine hohe Bedeutung zu. Führung verteilt sich auf unterschiedliche Rollen, als fachlicher Impulsgeber (z. B. in der Funktion eines Product Owners) oder methodischer Coach und Mediator bei Konflikten (z. B. in der Funktion eines Scrum Masters). Nicht Hierarchie, sondern die konkrete Lösung von Aufgaben und Problemen im Netzwerk von Fachexpert*innen steht im Vordergrund. Das Umsetzungsteam regelt Prioritäten und die Umsetzung von Maßnahmen eigenverantwortlich.

\subsection{Zur Historie von Führungstheorien und -ansätzen}

Warum in einem Kapitel über Führungsstile und -ansätze erst an dieser Stelle auf die Historie eingegangen wird und nicht bereits zu Beginn? Zum einen, weil es nicht Ziel ist, das wiederzugeben, was zahlreichen Fachpublikationen bereits umfangreich gelungen ist. Zum anderen, weil dieses Kapitel im Rückblick zeigen soll, dass viele der vorgenannten Führungsaspekte trotz modernem Anstrich nicht gänzlich neu sind, sondern ihre Ursprünge und Grundphilosophien bereits einige Jahre bzw. Jahrzehnte zurückliegen.

Aber worauf sollte man eingehen, wenn man sich mit der Historie von Führungstheorien und -ansätzen auseinandersetzen möchte? Und wo sollte man anfangen?

Die ältesten Führungstheorien setzen die Führungsperson in den Mittelpunkt des Interesses. Hieraus entwickelten sich die Führungsstil-Ansätze und Theorien zur situativen Führung.

In den 60er-Jahren wurden im deutschsprachigen Raum Führungstheorien entwickelt, die sich nicht mehr nur auf die Führungskraft und ihr Verhalten beziehen, sondern versuchen, die Organisation als Ganzes, als System zu erfassen. Als bekanntestes Beispiel dient das St. Galler Managementkonzept und der von dem ös- 
terreichischen Wirtschaftswissenschaftler und Unternehmensberater, Fredmund Malik, daraus weiterentwickelte Ansatz der erlernbaren, wirksamen Führung.

In den 70er-Jahren formten sich Ansätze, in denen die Beziehung zwischen Führenden und Geführten ins Zentrum der Betrachtung rückt, wie beispielsweise Servant Leadership, die Leader-Member-Exchange (LMX)-Theorie und Team Leadership.

Es existieren motivations-, machtorientierte und psychologische Ansätze von Führung. Nicht zuletzt dürfen die Management by-Ansätze (Management by Objectives, Management by Delegation, Management by Exception, Management by Decision Rules) nicht außer Acht gelassen werden, will man sich einen umfangreichen Überblick verschaffen.

Wie bereits erwähnt, werden diese Theorien und Ansätze in diesem Buch nicht aufgearbeitet, da es bereits zahlreiche ausgezeichnete Publikationen hierzu gibt (z. B. Stippler et al. 2011: Führung - Überblick über Ansätze, Entwicklungen, Trends).

Eine grafisch interessant aufbereitete Übersicht zu Führungstheorien bietet HRM INSPIRATION (2017) in ihrer Streetmap Leadership.

Dennoch kann ein Aufgreifen der wissenschaftlichen und praxisorientierten Historie von Führung nicht gänzlich außer Acht gelassen werden, wenn es darum geht, die Wirkungsmittel erfolgreicher Führung zu hinterfragen.

Provokant könnte man sogar die Frage stellen:

\section{Fragen}

Braucht es wirklich neue Führungsansätze oder sind die eigentlichen Kompetenzen, die eine Führungskraft mitbringen sollte, nicht seit jeher die gleichen, nur in einem sich veränderten Umfeld immer wieder neu orchestriert?

Einem Ansatz soll daher etwas mehr Aufmerksamkeit gewidmet werden, dessen Begründer bereits früh viele Entwicklungen in der Unternehmensführung vorhergesehen hat.

Bereits 1970 formulierte der Management-Vordenker Peter F. Drucker, ehemals Professor an der Claremont Graduate University, acht Prinzipien wirksamer und effektiver Führung:

1. Die richtigen Ziele setzen: Fragen, was zu tun ist, Prioritäten festlegen und diese strikt einhalten.

2. Prüfen, was gut und richtig für das Unternehmen ist: Die Interessen der wichtigsten Stakeholder in Entscheidungen mit einbeziehen. 
3. Einen Aktionsplan aufstellen: Sich den gewünschten Ergebnissen nähern, Ideen in die Tat umsetzen und einen Umsetzungsplan erstellen. Dieser sollte öfters überarbeitet werden.

4. Handeln und Verantwortung übernehmen: Der Entscheidungsfindung, der Kommunikation, den Chancen und Risiken sowie den Besprechungen besondere Aufmerksamkeit widmen, um Pläne in konkrete Maßnahmen zu übersetzen.

5. Stimmige Kommunikation sicherstellen: Alle müssen den Umsetzungsplan verstehen. Klären, dass jede/r weiß, welche Aufgaben er/sie erledigen muss und welchen Beitrag er/sie damit zur Zielerreichung des Unternehmens leistet.

6. Sich auf Chancen konzentrieren: Jede Form von Wandel als Chance betrachten, statt als Bedrohung. Innerhalb und außerhalb des Unternehmens systematisch Ausschau nach Veränderungen halten.

7. Eine effiziente Sitzungskultur schaffen: Dafür sorgen, dass die Zusammenkünfte effektive Arbeitstreffen sind und nicht aufgeregte Diskussionsrunden. Eine effektive Nachbereitung der Sitzung sicherstellen.

8. Sich als Teil des Hauses verstehen: Der „Wir-Form“ den Vorzug vor der „IchForm" geben. Führung wirkt vor allem dann, wenn die Menschen im Unternehmen der Führungskraft vertrauen.

Bonusprinzip: Erst zuhören, dann sprechen.

Anhand der ersten beiden Prinzipien erwerben sich Führungskräfte das erforderliche Wissen, das sie brauchen, um wirksam zu sein. Die Prinzipien drei bis sieben stehen für ein effektives Handeln, das auf dem erworbenen Wissen aufsetzt. Das letzte Prinzip bildet das Grundverständnis ab, das eine Wirksamkeit von Führung überhaupt erst möglich macht.

Warum diese Prinzipien zeitlos sind und auch heute noch eine hohe Aussagekraft besitzen?

Nach Druckers Einschätzung ist die Erhöhung der Produktivität von Wissensarbeiter*innen das wichtigste Ziel, welches das Management im 21. Jahrhundert erreichen muss (1959).

Er verweist damit weit vor dem Beginn des heutigen Digitalisierungs- und Innovationszeitalters auf die Bedeutung eines optimalen Informationssystems, die Notwendigkeit von Innovationen - auch, um die qualifizierten, sachkundigen Mitarbeiter*innen anzuziehen - und das Erfordernis der Selbstautonomie von Wissensarbeiter*innen (Drucker 2004). 
Und selbst das Thema nachhaltige Unternehmensführung scheint Drucker nicht außer Acht zu lassen, wenn er sich im Anschluss an die Fragen „Was ist zu tun?“ und „Wo liegen meine Prioritäten?“ über die folgenden Fragen mit möglichen Hemmnissen auseinandersetzt (2004).

- Ist unser Vorgehen ethisch einwandfrei?

- Ist unser Vorgehen legal?

- Ist unser Vorgehen unternehmensintern akzeptabel?

- Ist unser Vorgehen mit unseren Ideen, unseren Werten und unserer Unternehmenspolitik kompatibel?

Die Bedeutung der Ansätze Druckers hebt auch Professor Fredmund Malik (1998) hervor, wenn er schreibt:

Man muss nicht alle Auffassungen Druckers teilen, aber man braucht sehr gute Argumente, um sie abzulehnen. Ignorieren kann man sie jedenfalls nicht, wenn man ernst genommen werden will.

Die Prinzipien wirksamer Führung haben heute noch die gleiche Gültigkeit, wie vor 50 Jahren. Tab. 4.5 versucht einen groben Brückenschlag zwischen den Prinzipien Druckers und den Anforderungen an eine moderne Arbeitswelt.

Die Rahmenbedingungen für Führung haben sich einschneidend verändert. Wenden Führungskräfte von Banken und Sparkassen diese Prinzipien jedoch auch heute bei der strategischen Ausrichtung des Hauses oder im operativen Führungsalltag konsequent an, werden sie auch diesen Rahmenbedingungen gerecht.

Dass dies durchaus auch von den Mitarbeiter*innen so gesehen wird, zeigen Köhler und Hamm (2019) in ihrer Studie „Wettbewerbsfaktor Mensch“. Dort stellen sie unter dem provokanten Titel „New Work? No way!“ in einer repräsentativen Erhebung mit 1.100 Mitarbeitenden unterschiedlicher Unternehmen, Branchen, Hierarchien und Altersgruppen in Deutschland überraschende Ergebnisse vor.

Statt agiler Projektteams (Agilität und Innovation), selbstorganisiertem Arbeiten (operative Freiheit) oder New Leadership Ansätzen (Offenheit, Toleranz, Fehlerkultur, Führung als Coach) lösen vor allem authentische Führung mit Vorbildfunktion, Fürsorge im Sinne beruflicher Weiterentwicklungsmöglichkeiten und ein sicherer Arbeitsplatz Begeisterung bei Mitarbeiter*innen aus.

Laut der Studie sind Glaubwürdigkeit, Orientierung, klare Zielvorgaben und natürlich auch Handlungsspielraum innerhalb gesteckter Grenzen gefragt. Die vornehmliche Aufgabe der Führungskräfte sollte es sein, die Unternehmensstrategie zu vermitteln und mit ihrem Team einen sinnvollen Beitrag dazu zu leisten. So die Erwartungen der Mitarbeiter*innen, oder wie Köhler es formuliert: 
Tab. 4.5 Prinzipien nach Drucker und ihre Anwendung in der aktuellen Arbeitswelt

\begin{tabular}{|c|c|}
\hline Prinzip nach Drucker & Anwendung in der aktuellen Arbeitswelt \\
\hline Richtige Ziele setzen. & $\begin{array}{l}\text { Erfährt gerade bei der Führung virtueller oder } \\
\text { selbstverantwortlicher Teams eine hohe Bedeutung, da dies } \\
\text { insbesondere der Eigenverantwortlichkeit und Selbststeuerung } \\
\text { jedes einzelnen Teammitgliedes bedarf. }\end{array}$ \\
\hline $\begin{array}{l}\text { Prüfen, was gut und } \\
\text { richtig für das } \\
\text { Unternehmen ist. }\end{array}$ & $\begin{array}{l}\text { Ein wichtiges Element agiler Arbeitsformen, wie z. B. Design } \\
\text { Thinking, aber auch erfolgreicher Changeprozesse. }\end{array}$ \\
\hline $\begin{array}{l}\text { Einen Aktionsplan } \\
\text { erstellen. }\end{array}$ & $\begin{array}{l}\text { Insbesondere bei Führung auf Sicht, steigender Komplexität und } \\
\text { stetigen Veränderungen relevant. }\end{array}$ \\
\hline $\begin{array}{l}\text { Handeln und } \\
\text { Verantwortung } \\
\text { übernehmen. }\end{array}$ & $\begin{array}{l}\text { Bindet Mitarbeiter*innen im Zuge einer zunehmenden } \\
\text { Digitalisierung und Agilität mehr in Führungsverantwortung und } \\
\text { Führungskräfte mehr in Arbeitsprozesse ein. }\end{array}$ \\
\hline $\begin{array}{l}\text { Stimmige } \\
\text { Kommunikation } \\
\text { sicherstellen. }\end{array}$ & $\begin{array}{l}\text { Sinnhaftigkeit ist nach wie vor eines der zentralen Elemente der } \\
\text { Mitarbeitermotivation und -bindung. Wirkt zudem der Gefahr der } \\
\text { Informationsüberfrachtung in einer zunehmend digitalen } \\
\text { Unternehmenskommunikation entgegen und schafft } \\
\text { Verbindlichkeit. }\end{array}$ \\
\hline $\begin{array}{l}\text { Sich auf Chancen } \\
\text { konzentrieren. }\end{array}$ & Schafft ein positives Veränderungsumfeld: Sog statt Druck. \\
\hline $\begin{array}{l}\text { Eine effiziente } \\
\text { Sitzungskultur } \\
\text { schaffen. }\end{array}$ & $\begin{array}{l}\text { Insbesondere durch die Nutzung neuer Möglichkeiten und Tools } \\
\text { aus der digitalen und agilen Arbeitswelt. }\end{array}$ \\
\hline $\begin{array}{l}\text { Sich als Teil des } \\
\text { Hauses verstehen. }\end{array}$ & Stellt Authentizität und Glaubwürdigkeit sicher. \\
\hline
\end{tabular}

Eigene Darstellung

Viel wichtiger als reflexartig modischen Buzzwords zu folgen, ist es, sich in den Unternehmen wieder mit dem zu beschäftigen, was echte Führung ausmacht.

Die Aussagen der ,alten Meister“, wie Drucker oder Malik, haben somit auch heute noch Gewicht. So gehen beide in ihren Überlegungen davon aus, dass Führung erlernt werden kann. Aber ist dem wirklich so? Mit dieser Frage soll sich das folgende Kapitel auseinandersetzen.

\section{Fazit aus diesem Abschnitt}

Tradierte Ansätze von Führung haben nach wie vor ihre Berechtigung. Übersetzt man sie in die moderne Welt bzw. wendet man sie auf die aktuellen Anforderungen und Rahmenbedingungen an, so sind ihre Kernmerkmale ebenso wirkungsvoll, wie die neuerer, moderner Ansätze. 


\section{Reflexionsfragen zum Kapitel}

- Welche Elemente neuer Führungsstile/-ansätze passen am besten zur gewünschten Führungskultur der Bank/Sparkasse? Zahlen diese auf das Unternehmensleitbild und die Strategie des Hauses positiv ein? Auf was kommt es bei Führung wirklich an?

- Bringen die Führungskräfte des Hauses die erforderlichen Persönlichkeitsmerkmale mit, um das gewünschte Führungsverhalten in der Praxis umzusetzen?

- Wie gelingt es, Emotionalität angemessen in den Führungsalltag einfließen $\mathrm{zu}$ lassen?

- Woran lassen sich Authentizität und Vertrauen im Führungsverhalten konkret festmachen? Wie wird beides für die Mitarbeiter*innen erlebbar?

- Wie ist der digitale Reifegrad der einzelnen Führungskräfte? Wie sind die damit verbundenen Zielvorstellungen? Wie werden Führungskräfte dahingehend entwickelt?

- Wie stellt Führung Nähe bei der Führung von Mitarbeiter*innen auf Distanz sicher?

- Welche Maßnahmen/Regelungen werden getroffen, um eine effiziente Teamarbeit im virtuellen Raum bzw. auf Distanz sicher zu stellen?

- In welchen Teilbereichen der Bank/Sparkasse machen agile Arbeitsmethoden Sinn?

- Wie werden die damit betrauten Führungskräfte und Mitarbeiter*innen auf die agile Arbeit vorbereitet?

- Wie geht man an den Schnittstellen zur Hierarchie der Bank/Sparkasse miteinander um, ohne die agilen Arbeitsprozesse in Frage zu stellen bzw. auszuhebeln?

\section{Literatur}

Akin N, Rumpf J (2013) Führung virtueller Teams. Gruppendynamik \& Organisationsberatung 44(4):373-387

Alberts DS, Hayes RE, Honekamp W (Übersetzer) (2009) Power to the edge. Re Di Roma, Remscheid

Appelo J (2010) Management 3.0. Leading Agile Developers, Developing Agile Leaders. Addison-Wesley Professional, Upper Saddle River

Avolio BJ, Gardner WL, Walumbwa FO, Luthans F, May DR (2004) Unlocking the mask: a look at the process by which authentic leaders impact follower attitudes and behaviors. Leadersh Q 15(6):801-823

Bass BM (1985) Leadership and performance beyond expectations. Free Press, New York 
Bass BM, Avolio BJ (1990) Developing transformational leadership: 1992 and beyond. J Work Organ Psychol 8(1):9-32

Buhse W (2014) Management by Internet - Neue Führungsmodelle für Unternehmen in Zeiten der digitalen Transformation. Plassen, Kulmbach

Burns JM (1978) Leadership. Harper and Row, New York

Capgemini Invent (2015) Digital Leadership-Führungskräfteentwicklung im digitalen Zeitalter. http://www.capgemini.com/consulting-de/wp-content/uploads/sites/32/2017/08/14-10-16_ digital_leadership_v11_web_17102016.pdf. Zugegriffen am 14.11.2019

Conger JA, Kanungo R (1998) Charismatic leadership in organizations. SAGE Publications, San Francisco

Drucker PF (1959) Landmarks of tomorrow. Harper, New York

Drucker PF (2004) Concept of the corporation. Harper, New York

Eberhardt D, Majkovic AL (2015) Die Zukunft der Führung. Eine explorative Studie zu den Führungsherausforderungen von morgen. Springer Fachmedien, Wiesbaden

Fajen A (2017) Erfolgreiche Führung multikultureller virtueller Teams. Wie Führungskräfte neuartige Herausforderungen meistern. Springer Gabler, Wiesbaden

Fox R (2017) Bionische Unternehmensführung. Mitarbeitermotivation als Schlüssel zu Innovation, Agilität und Kollaboration. Springer Gabler, Wiesbaden

Freyth A, Baltes G (2017) Veränderungsintelligenz auf individueller Ebene Teil 2: Persönliche Agilität und agiler führen. Diagnose und Stärkung von persönlicher Agilität, veränderte Führungsrolle im agilen Kontext. In: Baltes G, Freyth A (Hrsg) VeränderungsIntelligenz. Springer Fachmedien, Wiesbaden

Furtner M (2016) Effektivität der transformationalen Führung. Springer Gabler, Wiesbaden

Gabler Wirtschaftslexikon (2019) Matrixorganisation. https://wirtschaftslexikon.gabler.de/ definition/matrixorganisation-39659. Zugegriffen am 19.11.2019

Gardner WL, Avolio BJ, Luthans F, May DR, Walumbwa F (2005) „Can you see the real me?" A self based model of authentic leader and follower development. Leadersh Q 16(3):343-372

Geschwill R, Nieswandt M (2016) Laterales Management. Das Erfolgsprinzip für Unternehmen im digitalen Zeitalter. Springer Fachmedien, Wiesbaden

Grote G, Manchen-Spörri S, Springall L (2004) Telemanagement: Notwendigkeit für ein komplexes Verhaltensrepertoire. Zeitschrift für Arbeitsforschung, Arbeitsgestaltung und Arbeitspolitik 13:48-60

Harms PD, Credé M (2010) Emotional intelligence and transformational and transactional leadership: a meta-analysis. J Leadersh Org Stud 17(1):5-17

Häusling A, Römer E, Zeppenfeld N (2017) Praxisbuch Agilität. Tools für Personal und Organisationsentwicklung. Haufe, Freiburg

Hays (2016) HR-Report 2017 - Schwerpunkt Kompetenzen für eine digitale Welt. https:// www.hays.de/personaldienstleistung-aktuell/studie/hr-report-2017. Zugegriffen am 08.12 .2019

Hofert S (2017) Agiler Führen. Einfache Maßnahmen für bessere Teamarbeit, mehr Leistung und höhere Kreativität. Springer Gabler, Wiesbaden

HRM INSPIRATION (2017) Streetmap leadership. https://4pleadership.wordpress. com/2017/08/26/eine-uebersicht-der-fuehrungstheorien/. Zugegriffen am 27.11.2019

Köhler W, Hamm I (2019) Wettbewerbsfaktor Mensch - Wie man durch Mitarbeiterbegeisterung und moderne Führung Mehrwert schafft. Vortrag zu den Ergebnissen der Studie an der Hochschule Darmstadt am 12. November 2019 
Korn HP (2016) Erfolgreiche Führung war schon immer agil! In: Geramanis O, Hermann K (Hrsg) Führen in ungewissen Zeiten. Springer Gabler, Wiesbaden

Kronawitter E (2013) Führen ohne Druck. Springer Gabler, Wiesbaden

Lasnia M, Nowotny V (2018) Agile Evolution. Eine Anleitung zur agilen Transformation. Business Village, Göttingen

Malik F (1998) Die Welt des Peter Drucker Anmerkungen zum gleichnamigen Buch von Jack Beatty. http://www.druckersociety.at/index.php/peterdruckerhome/commentaries/ prof-dr-fredmund-malik. Zugegriffen am 27.11.2019

Oestereich B, Schröder C (2017) Das kollegial geführte Unternehmen. Vahlen, München

Petry T (2019) Digital Leadership: Erfolgreiches Führen in Zeiten der Digital Economy. Haufe, Freiburg

Philipp AF (2010) Die Kunst ganzheitlichen Führens. Ein Praxishandbuch für den LeaderAlltag. Literatur-VSM, Wolkersdorf

Radermacher I (2018) Digitalisierung selbst denken. Eine Anleitung mit der die Transformmation gelingt. BusinessVillage, Göttingen

Remdisch S (2005) Managing virtual teams: The importance of distance leadership. Führung auf Distanz. Universität Lüneburg. http://www2.leuphana.de/distanceleadership/download/dl_praesentation-uni.pdf. Zugegriffen am 10.11.2019

Rochus Mummert (2013) Management 2.0: Drei von vier virtuellen Teams scheitern. http:// www.rochusmummert.com/downloads/news/67_130416_rochus_mummert_virtuelle_ teams.pdf. Zugegriffen am 17.11.2019

Scheller T (2017) Auf dem Weg zur agilen Organisation. Wie Sie ihr Unternehmen dynamischer, flexibler und leistungsfähiger gestalten. Vahlen, München

Schiefer G, Nitsche H (2019) Die Rolle der Führungskraft in agilen Organisationen. Springer Fachmedien, Wiesbaden

Slogar A (2018) Die agile Organisation: Wo anfangen? Wie Mitarbeiter begeistern? Wie Strukturen und Strategien anpassen? Carl Hanser, München

Sprenger RK (2017) Transformationale Führung - Was will sie? Wie geht sie? In: Walter J, Böckenholt I, Diestel S (Hrsg) HR-Exzellenz. Innovative Ansätze in Leadership und Transformation. Springer Gabler, Wiesbaden

Stippler M, Moore S, Rosenthal S, Dörffer T (2011) Führung - Überblick über Ansätze, Entwicklungen, Trends. Bertelsmann Stiftung, Gütersloh

Wächter G (2019) Digital Leadership - Neuer Wein in alten Schläuchen? In: Fürst RA (Hrsg) Gestaltung und Management der digitalen Transformation. Springer Fachmedien, Wiesbaden

WagnerDJ(2018)DigitalLeadership,Kompetenzen-Führungsverhalten-Umsetzungsempfehlungen. Springer Gabler, Wiesbaden

Wald PM (2014) Virtuelle Führung. In: Lang R, Rybnikova I (Hrsg) Aktuelle Führungstheorien und -konzepte. Springer Fachmedien, Wiesbaden

Wolf H (2003) Partizipatives Management - was bleibt? Expertise für die Hans-BöcklerStiftung. http://www.sofi-goettingen.de/fileadmin/Harald_Wolf/Material/Wolf_Partizipatives_Management.pdf. Zugegriffen am 17.11.2019

Zeb (2018) Die Entwicklung des deutschen Bankenmarkts inkl. Ausblick auf 2019. https:// bankinghub.de/banking/research-markets/bankenmarkt-2018. Zugegriffen am 17.11.2019 